\title{
ANALYTICAL AND COMPUTATIONAL METHODS FOR TWO-PHASE FLOW WITH SOLUBLE SURFACTANT*
}

\author{
KUAN XU ${ }^{\dagger}$, M. R. BOOTY ${ }^{\dagger}$, AND M. SIEGEL $^{\dagger}$
}

\begin{abstract}
A hybrid method is used to determine the influence of surfactant solubility on twophase flow by solution of a reduced transition layer equation near a fluid interface in the limit of large bulk Péclet number. The method is applied to finding the evolution of a drop of arbitrary viscosity that is deformed by an imposed linear strain or simple shear flow. A semianalytical solution of the transition layer equation is given that expresses exchange of surfactant between its bulk and interfacial forms in terms of a convolution integral in time. Results of this semianalytical solution are compared with the results of a spatially spectrally accurate numerical solution. Although both the hybrid method and its semianalytical solution are valid in three dimensions, the two-dimensional context of this study allows additional validation of results by comparison with those of conformal mapping techniques applied to inviscid bubbles.
\end{abstract}

Key words. interfacial flow, two-phase flow, soluble surfactant

AMS subject classifications. 76D45, 65N80, 35Q35

DOI. $10.1137 / 120881944$

1. Introduction. Surfactant is a generic term for a surface active agent that reduces the surface tension between immiscible liquids. Surfactants are in widespread use in a variety of applications in the chemical, petrochemical, agrochemical, and biotechnology industries, and they are also used in food processing and in many commercial and household products. Many types of surfactant are manufactured or synthesized, but they also occur naturally and are produced by some microorganisms. Surfactants are often used as detergents, dispersants, or emulsifiers, foaming, or wetting agents. An overview of their use and properties is given in, for example, [1].

The action of surfactants results from the amphiphilic structure of a surfactant molecule, which has a long hydrophobic tail and a relatively small hydrophilic or polar head. In a fluid mixture it will therefore typically be present at the interface between an aqueous phase and a dissimilar phase, for example a gas or a hydrocarbon liquid. A surfactant is, however, soluble to some extent in either the continuous or the dispersed phase of a two-phase mixture, so that it can be exchanged between the bulk fluid and the fluid interface. At sufficiently low concentrations, surfactant in the bulk flow behaves as a solute of individual molecules that diffuse very slowly due to their relative lack of mobility and high molecular weight, which is typically in the range of 200-2,000 amu. At higher concentrations, at and above the critical micelle concentration, surfactant molecules that are present in the bulk can also occur as multimolecule aggregates or micelles. This can influence the effects of surfactant solubility and is not considered here.

We develop a model for the study of surfactant solubility effects in two-phase flow that was introduced in [2]. This utilizes the small diffusion coefficient of the bulk

${ }^{*}$ Received by the editors June 21, 2012; accepted for publication (in revised form) November 26, 2012; published electronically February 26, 2013. This work was supported by National Science Foundation grants DMS-0708977 and DMS-1009105.

http://www.siam.org/journals/siap/73-1/88194.html

$\dagger$ Department of Mathematical Sciences and Center for Applied Mathematics and Statistics, New Jersey Institute of Technology, Newark, NJ 07102 (kuan.xu@njit.edu, booty@njit.edu, misieg@njit. edu). 
surfactant concentration, or equivalently its large bulk Péclet number $P e$, to form a leading order, local reduction of the dynamics of soluble surfactant in a narrow transition or boundary layer that forms immediately adjacent to the fluid interface. This type of approximation is familiar in the context of matched asymptotic expansions, and it is intended to resolve accurately the exchange of surfactant between its bulk (or dissolved) and interfacial (or adsorbed) forms in the limit $P e \rightarrow \infty$. Away from the transition layer, the bulk concentration is taken to be spatially uniform and effectively diffusion-free.

The study focuses on the canonical example of a single drop or bubble that is deformed by an imposed flow. This has been the subject of much work since the early systematic study by Taylor appeared in 1934 [3]. Examples of experimental studies of drop deformation and breakup, with and without surfactant, have been given by Grace [4], Bentley and Leal [5], de Bruijn [6], and Janssen, Boon, and Agterof [7, 8]. Numerical studies have been given by, for example, Stone and Leal [9], Milliken, Stone, and Leal [10], and Bazhlekov, Anderson, and Meijer [11], and recent reviews of the literature have been given by Stone [12], Eggers [13], Quéré [14], and Basaran [15]. A notable example of drop dynamics in the presence of surfactant is the phenomenon of tip-streaming, which is discussed in many of these references and has been the specific subject of recent numerical [16] and experimental [17] investigations.

A major theme of this study is the presentation and validation of an analytical, Green's function-based method for solving the transition layer equation. Derivation of the result appears in section 5, which gives the net flux of surfactant entering or leaving each point of the interface in the form of a convolution integral over time. The surfactant exchange flux appears as a source term in the equation for conservation of the surface concentration of surfactant. The method can be thought of as meshfree, in the sense that no spatial mesh needs to be introduced in the direction normal to the interface to solve the transition layer equation for bulk surfactant concentration. Instead, the spatial structure of the transition layer in the normal direction is contained in the convolution integral, while the spatial structure in the direction tangential to the interface enters only parametrically, via a Lagrangian fluid marker on the interface.

We confine our attention to a 2D (two-dimensional) Cartesian geometry and the zero Reynolds number Stokes flow limit for incompressible flow of two immiscible Newtonian fluids. The infinite Péclet number limit transition layer equation and the mesh-free Green's function-based method can readily be extended to three dimensions and nonzero Reynolds number, but the $2 \mathrm{D}$ geometry allows an additional means of validation, especially in the limit when the viscosity of the interior or drop fluid is zero. In this limit, conformal mapping techniques have been applied and show that for a 2D inviscid drop that is initially circular or elliptical, when placed in either a pure strain or a linear shear flow, the drop shape remains elliptical for all time with a time-dependent eccentricity. This has been shown in the absence of surfactant [18], and with surfactant that is either insoluble [19] or soluble [2], and rests on the exact truncation of the conformal map to a Joukowski transformation.

Here the interior or drop fluid viscosity is arbitrary; that is, the ratio of the interior to exterior fluid viscosity, which is denoted by $\lambda$, is such that $\lambda \geq 0$. For $2 \mathrm{D}$ Stokes flow the solution for the fluid velocity and pressure can be written in terms of a stream function that satisfies a boundary value problem for the biharmonic equation, which can in turn be expressed in terms of the solution of a Sherman-Lauricella integral equation. This representation is perhaps more familiar in the context of classical 2D problems of elasticity (see, for example, [20,21]), but it has also been applied to two- 
phase Stokes flow; see, for example, [28] and other references given at the beginning of section 3. When the interior fluid is viscous, $\lambda>0$, the Sherman-Lauricella formulation is more computationally efficient as a means of solving the biharmonic equation than conformal mapping techniques. However, it is not apparent or built into the Sherman-Lauricella formulation that the exact elliptical drop profile will be retained in the inviscid limit when $\lambda=0$. The fact that this is observed provides validation that the underlying flow solver, together with the technique for including insoluble surfactant and soluble surfactant effects via either a mesh-based or mesh-free method, has been implemented correctly.

The formulation of the problem is given in section 2. The Sherman-Lauricella integral equation for the underlying fluid flow solver is set up in section 3. The mesh-based method for solving the transition layer equation is given in section 4, and numerical results that compare this with the Green's function-based method of section 5 are given in section 6 . Concluding remarks appear in section 7 .

2. Formulation. We consider a single fluid drop in an immiscible exterior fluid. Both fluids are Newtonian, and the flow is incompressible. The drop has undeformed radius $a_{0}$ and is deformed by an imposed linear flow. The exterior fluid has molecular viscosity $\mu$ and occupies the region $\Omega$, while the interior or drop fluid has molecular viscosity $\mu^{i}=\lambda \mu$ and occupies the complementary region $\Omega^{i}$. Throughout this study, an $i$ superscript denotes a quantity in the interior or drop region. The interface $\partial \Omega$ has surface tension $\sigma_{0}$ in the absence of surfactant, which is taken as a reference value. All lengths are made nondimensional by the undeformed drop radius $a_{0}$, velocities are made nondimensional by the capillary velocity in the absence of surfactant $U=\sigma_{0} / \mu$, time is made nondimensional by the scale $\tau=a_{0} / U$, and the fluid pressure is made nondimensional by the drop capillary pressure $\sigma_{0} / a_{0}$.

This study focusses on the influence of soluble surfactant on the drop's shape and dynamics. The surface surfactant concentration is made nondimensional by the maximum monolayer packing concentration $\Gamma_{\infty}$, and the bulk surfactant concentration is made nondimensional by a uniform far-field reference value $C_{\infty}$. The surfactant has bulk diffusion coefficient $D$ and surface diffusion coefficient $D_{s}$, both of which are taken to be small. Surfactant can transfer or exchange between its adsorbed form on the interface and its dissolved form in the bulk immediately adjacent to the interface, with a desorption rate $\kappa_{d}$ and adsorption rate $\kappa_{a}$.

The drop and exterior fluid have the same density, so that the drop is neutrally buoyant, and gravitational effects are absent. The geometry is $2 \mathrm{D}$, and some notation is shown in the schematic of Figure 2.1. On $\partial \Omega$ the unit normal vector $\boldsymbol{n}$ points from $\Omega^{i}$ to $\Omega$, the unit tangent vector $s$ points in the direction such that the interior $\Omega^{i}$ is to the right as $\partial \Omega$ is traversed clockwise, and $\theta$ is the angle measured counterclockwise positive from the positive $x$-axis to $s$.

In the zero Reynolds number or Stokes flow limit the nondimensional governing equations for momentum and mass transport are

$$
\begin{aligned}
\nabla^{2} \boldsymbol{u} & =\nabla p, & \nabla \cdot \boldsymbol{u} & =0, & & \boldsymbol{x} \in \Omega, \\
\lambda \nabla^{2} \boldsymbol{u}^{i} & =\nabla p^{i}, & \nabla \cdot \boldsymbol{u}^{i} & =0, & & \boldsymbol{x} \in \Omega^{i},
\end{aligned}
$$

where $\boldsymbol{u}$ is the velocity of the exterior fluid, $p$ is its pressure, and throughout an $i$ superscript denotes the corresponding quantity for the interior or drop fluid.

The fluid velocity is continuous across the interface, that is,

$$
\boldsymbol{u}(\boldsymbol{x}, t)=\boldsymbol{u}^{i}(\boldsymbol{x}, t), \quad \boldsymbol{x} \in \partial \Omega,
$$




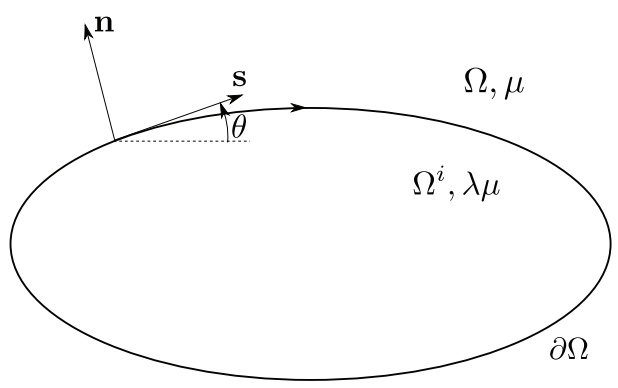

FIG. 2.1. A drop with viscosity $\lambda \mu$ is enclosed by fluid with viscosity $\mu$. The unit normal $\boldsymbol{n}$ on the interface $\partial \Omega$ points from $\Omega^{i}$ to $\Omega$, the unit tangent vector $\boldsymbol{s}$ points in the direction shown, and $\theta$ is the angle measured counterclockwise positive from the positive $x$-axis to $\boldsymbol{s}$. The curvature $\kappa=-\frac{\partial \theta}{\partial s}$ is positive where the shape is convex.

and if $\boldsymbol{x}$ is the position of a material particle at the interface, then it satisfies

$$
\frac{d \boldsymbol{x}}{d t}=\boldsymbol{u}(\boldsymbol{x}, t), \quad \boldsymbol{x} \in \partial \Omega .
$$

The evolution of the interface shape is determined by the component of its velocity along the normal, so that for any point $\boldsymbol{x}$ on the interface we have the kinematic condition

$$
\frac{d \boldsymbol{x}}{d t} \cdot \boldsymbol{n}=\boldsymbol{u} \cdot \boldsymbol{n} \equiv u_{n}, \quad \boldsymbol{x} \in \partial \Omega .
$$

In the inviscid limit $\lambda=0$, the interior pressure $p^{i}(t)$ is a function of time $t$ alone, independent of position, and the interior velocity $\boldsymbol{u}^{i}$ is not specified. The condition of continuity of velocity across $\partial \Omega$ is then dropped, but the kinematic condition still holds.

The difference in the fluid stress across $\partial \Omega$ is equal to the net stress due to interfacial surface tension, which leads to the stress-balance boundary condition

$$
-\left(p-p^{i}\right) \boldsymbol{n}+2\left(\boldsymbol{e}-\lambda \boldsymbol{e}^{i}\right) \cdot \boldsymbol{n}=\sigma \kappa \boldsymbol{n}-\nabla_{s} \sigma, \quad \boldsymbol{x} \in \partial \Omega .
$$

See, for example, [22]. Here $\boldsymbol{e}$ is the rate-of-strain tensor with entries $e_{i j}=\frac{1}{2}\left(\partial_{x_{i}} u_{j}+\right.$ $\left.\partial_{x_{j}} u_{i}\right), \kappa$ is the local curvature of the interface, and $\sigma$ is the surface tension, which depends on the adsorbed or surface concentration of surfactant $\Gamma$ through a surface equation of state $\sigma=\sigma(\Gamma)$. The presence of surfactant on an interface reduces its surface tension, and two specific choices for an equation of state that are widely used are the equation derived from the Langmuir adsorption isotherm, which is referred to as the Frumkin equation, and a linearized version for small surfactant concentration. These are

$$
\sigma= \begin{cases}1+E \ln (1-\Gamma) & \text { the Frumkin equation of state } \\ 1-E \Gamma & \text { a linearized equation of state }\end{cases}
$$

where $E=R T \Gamma_{\infty} / \sigma_{0}$ is the elasticity parameter, which is a dimensionless measure of the sensitivity of surface tension to adsorbed surfactant concentration [23]. We have chosen to use the linearized equation of state. The last term on the right-hand side of (2.4) is referred to as the Marangoni stress. Here $\nabla_{s}$ is the surface gradient operator, and the Marangoni stress is caused by the nonzero spatial gradient of the 
interfacial surface tension that occurs when the distribution of adsorbed surfactant on the interface is not spatially uniform.

This study focusses on surfactant that is soluble, so that surfactant is also present in its bulk or dissolved form away from the interface, where it has bulk concentration $C$ and is transported as a passive scalar. For simplicity, surfactant is taken to be soluble only in the external fluid region $\Omega$, so that the bulk concentration $C$ satisfies

$$
\frac{\partial C}{\partial t}+\boldsymbol{u} \cdot \nabla C=\frac{1}{P e} \nabla^{2} C, \quad \boldsymbol{x} \in \Omega,
$$

where $P e=U a_{0} / D$ is the bulk Péclet number, which is typically large in applications, and can be of the order of $10^{6}$ or more.

The surface concentration of surfactant, $\Gamma$, satisfies the conservation law

$$
\left.\frac{\partial \Gamma}{\partial t}\right|_{\xi}-\left.\frac{\partial \boldsymbol{X}}{\partial t}\right|_{\xi} \cdot \nabla_{s} \Gamma+\nabla_{s} \cdot\left(\Gamma \boldsymbol{u}_{s}\right)+\Gamma \kappa u_{n}=\frac{1}{P e_{s}} \nabla_{s}^{2} \Gamma+\left.J \boldsymbol{n} \cdot \nabla C\right|_{\partial \Omega}, \quad \boldsymbol{x} \in \partial \Omega
$$

see [24]. Here $\boldsymbol{u}_{s}$ is the projection of the fluid velocity onto the tangent plane at the interface, $u_{n}=\boldsymbol{u} \cdot \boldsymbol{n}$ is the normal component of velocity of the interface, $\boldsymbol{x}=\boldsymbol{X}(\boldsymbol{\xi}, t)$ is a parametric representation of the interface, and $P e_{s}=U a_{0} / D_{s}$ is the surface Péclet number. On the left-hand side of (2.7), the first two terms ensure that if the interface moves, then the time derivative of $\Gamma$ is taken in the direction $\boldsymbol{n}$ normal to the interface, while the next two terms account for the change in surfactant concentration due to advective flux along the interface and the change in local interfacial area caused by motion along the normal. The first term on the right-hand side represents the change in $\Gamma$ due to surface diffusion, which is usually neglected, while the second term accounts for the transfer or exchange of surfactant between its dissolved form in the bulk flow immediately adjacent to the interface and its adsorbed form on the interface. The parameter $J=D C_{\infty} / U \Gamma_{\infty}$ is a measure of the diffusive flux for exchange of surfactant between the bulk and interface relative to the advective flux of adsorbed surfactant on the interface.

Exchange of surfactant between the bulk phase neighboring the interface and the interface itself is a two-step process [1]. In the bulk, surfactant is transported relative to material on the interface by diffusion, while exchange between the bulk and the interface occurs via adsorption-desorption kinetics. The net rate of accumulation of surfactant on the interface is therefore equal to its rate of adsorption minus its rate of desorption and is also equal to the normal diffusive flux of bulk surfactant at the interface, so that

$$
\left.J \boldsymbol{n} \cdot \nabla C\right|_{\partial \Omega}=B i\left(\left.K(1-\Gamma) C\right|_{\partial \Omega}-\Gamma\right), \quad \boldsymbol{x} \in \partial \Omega .
$$

In (2.8), the dimensionless parameter $K=\kappa_{a} C_{\infty} / \kappa_{d}$ is an equilibrium partition coefficient, and the Biot number $B i=\kappa_{d} a_{0} / U$ is the ratio of the time scale of the flow $a_{0} / U$ to the time scale of the kinetic desorption process $\kappa_{d}^{-1}$. In this study we consider the limit $B i \rightarrow \infty$, which is realistic in applications when the drop size is around $0.1 \mathrm{~mm}$ or larger and is referred to as the diffusion-controlled regime. The boundary condition (2.8) then simplifies to the Dirichlet boundary condition

$$
\left.C\right|_{\partial \Omega}=\frac{\Gamma}{K(1-\Gamma)}, \quad x \in \partial \Omega,
$$

which is the equilibrium adsorption relation of the Langmuir isotherm. 
If the initial distribution of bulk surfactant is spatially uniform and its far-field value is constant in time, then

$$
C(\boldsymbol{x}, 0)=1 \text { for all } \boldsymbol{x} \in \Omega \text { and } C(\boldsymbol{x}, t) \rightarrow 1 \text { as }|\boldsymbol{x}| \rightarrow \infty \text { for } t>0 .
$$

The drop is deformed by an imposed linear far-field flow,

$$
\boldsymbol{u}^{\infty}=\left(\begin{array}{cc}
Q & B+G / 2 \\
B-G / 2 & -Q
\end{array}\right) \cdot \boldsymbol{x}+O\left(|\boldsymbol{x}|^{-2}\right) \quad \text { as }|\boldsymbol{x}| \rightarrow \infty,
$$

where the dimensionless parameters $(Q, B, G)$ are equal to their nondimensional counterparts $Q_{\infty}$, etc., times the time scale $a_{0} / U$; i.e., $(Q, B, G)=\left(a_{0} / U\right)\left(Q_{\infty}, B_{\infty}, G_{\infty}\right)$. Two familiar examples that will be discussed below are (i) a pure strain, hyperbolic flow, or uniaxial extension given by $\boldsymbol{u}^{\infty} \sim Q\left(x_{1},-x_{2}\right)$ when $B=G=0$, where $Q$ is the capillary number, and (ii) the linear shear flow given by $\boldsymbol{u}^{\infty} \sim G\left(x_{2}, 0\right)$ when $Q=0$ and $G=2 B$, where $G$ is the capillary number.

2.1. The infinite Péclet number limit. Equation (2.6) for $C$ is singularly perturbed in the limit $P e \rightarrow \infty$. This study follows the approach of matched asymptotic expansions taken in [2], where the exact evolution is approximated by its leading order form in a neighborhood of the interface $\partial \Omega$, which is referred to as a transition layer, while away from the transition layer the leading order concentration is uniform and equal to its far-field value $C=1$. A brief derivation of this approach is given here; further detail can be found in [2].

An intrinsic or surface-fitted orthogonal curvilinear coordinate system $(\xi, n)$ is used that is attached to $\partial \Omega$. It has origin $O^{\prime}$, while the laboratory Eulerian reference frame has origin $O$. The position vector $\boldsymbol{x}$ of a point $P$ relative to $O$ can be written in the two coordinate systems as $\boldsymbol{x}=\boldsymbol{X}(\xi, t)+n \boldsymbol{n}(\xi, t)$, where $\boldsymbol{X}=\overrightarrow{O P^{\prime}}$ and $P^{\prime}$ is the projection of $P$ onto $\partial \Omega$ in the direction of the unit normal $\boldsymbol{n}$, so that the interface $\partial \Omega$ has equation $\boldsymbol{x}=\boldsymbol{X}(\xi, t)$, as in (2.7).

The fluid velocity in the Eulerian frame $\boldsymbol{u}$, written in terms of its projection onto the tangent plane $\boldsymbol{u}_{t}$ and its component in the normal direction $u_{p}$, is $\boldsymbol{u}=\boldsymbol{u}_{t}+u_{p} \boldsymbol{n}$. The gradient operator is written similarly as $\nabla=\nabla_{t}+\boldsymbol{n} \partial_{n}$. In the intrinsic frame, (2.6) becomes

$$
\frac{\partial C}{\partial t}+\boldsymbol{v}_{t} \cdot \nabla_{t} C+v_{p} \frac{\partial C}{\partial n}=\frac{1}{P e} \nabla^{2} C .
$$

Here $\boldsymbol{v}_{t}=\boldsymbol{u}_{t}-\boldsymbol{U}_{s}$, where $\boldsymbol{U}_{s}$ is the velocity of $O^{\prime}$ relative to $O$ projected onto the tangent plane at $P$, and $v_{p}=u_{p}-u_{n}$. So, $\boldsymbol{v}_{t}$ is the fluid velocity relative to $O^{\prime}$ projected onto the tangent plane at $P$ and, since $u_{n}$ is the normal speed of the interface relative to $O, v_{p}$ is the normal component of the fluid velocity relative to $\partial \Omega$. As the interface is approached, $\boldsymbol{u}_{t}$ approaches the tangential interfacial fluid velocity $\boldsymbol{u}_{s}$, and $v_{p}$ tends to zero.

In the large $P e$ limit, $C$ can depend on a local normal coordinate $N$, where $n=\epsilon N, \epsilon=P e^{-1 / 2}$, and $N=O(1)$ as $\epsilon \rightarrow 0$ in the transition layer adjacent to the interface. Although $C=C(\xi, N, t ; \epsilon)$ within the layer, there is no mechanism available to support a similar separation of scales for the fluid velocity, so that $\boldsymbol{v}=\boldsymbol{v}_{t}+v_{p} \boldsymbol{n}=$ $\boldsymbol{v}(\xi, n, t ; \epsilon)$.

An equation for the evolution of $C$ within the layer that is exact in the limit $P e \rightarrow \infty$ is given by keeping only the leading terms in an expansion for small $\epsilon$. In this approximation, the tangential velocity $\boldsymbol{v}_{t}$ is replaced by its value on $\partial \Omega$,

$$
\boldsymbol{v}_{s}=\boldsymbol{u}_{s}-\boldsymbol{U}_{s},
$$


which is of order $O(1)$ except, for example, near stagnation points on the surface, and $\nabla_{t}$ is replaced by $\nabla_{s}$. Since $\partial \Omega$ is a fluid interface, the kinematic condition implies that $v_{p}$ vanishes on $\partial \Omega$, so that it is replaced by the first nonzero term of its Taylor expansion, $\left.\epsilon N \partial_{n} v_{p}\right|_{s}$, where the normal derivative $\left.\partial_{n} v_{p}\right|_{s}$ is evaluated on $\partial \Omega$. The small $O(\epsilon)$ estimate in size of this coefficient in (2.12) multiplies the normal gradient of $C$, which is approximated by $(1 / \epsilon) \partial_{N} C(\xi, N, t ; 0)$. In a similar way, the small diffusion coefficient of (2.12) is magnified by the Laplacian of $C$ within the layer, so that the right-hand side of (2.12) is approximated by $\partial_{N}^{2} C(\xi, N, t ; 0)$. The reduced equation that results for the evolution of a first approximation to $C$ within the layer is therefore

$$
\frac{\partial C}{\partial t}+\boldsymbol{v}_{s} \cdot \nabla_{s} C+\left.\frac{\partial v_{p}}{\partial n}\right|_{\partial \Omega} N \frac{\partial C}{\partial N}=\frac{\partial^{2} C}{\partial N^{2}}, \quad \boldsymbol{x} \in \Omega^{r},
$$

where $\Omega^{r}$ is the transition layer subdomain of $\Omega$. Consideration of higher order terms in the expansion implies that the error in approximating $C$ by the solution of this equation is of order $O(\epsilon)$ as $\epsilon \rightarrow 0$.

The large $P e$ limit implies that outside the transition layer $\left(\partial_{t}+\boldsymbol{u} \cdot \nabla\right) C=0$ to within $O(\epsilon)$, so that $C$ is constant on particle paths to the same order. The initial condition of (2.10), that $C(\boldsymbol{x}, 0)=1$ everywhere, implies that $C \equiv 1$ outside the transition layer for all time, so that (2.14) has initial, boundary, and matching conditions

$$
\begin{aligned}
& C(\xi, N, 0)=1,\left.\quad C\right|_{N=0}=\frac{\Gamma}{K(1-\Gamma)}, \\
& C(\xi, N, t) \rightarrow 1 \text { as } N \rightarrow \infty \text { for } t>0 .
\end{aligned}
$$

At a first glance, the coefficient $\left.\partial_{n} v_{p}\right|_{s}$ in (2.14) appears to require the evaluation of off-surface data to compute the normal derivative. However, from the incompressibility condition $\nabla \cdot \boldsymbol{u}=0$ written in the intrinsic frame

$$
\frac{1}{a} \frac{\partial}{\partial \xi}\left((1+n \kappa) u_{t}\right)+\frac{\partial}{\partial n}\left((1+n \kappa) u_{p}\right)=0,
$$

where $u_{t}$ is the magnitude of the tangential velocity $\boldsymbol{u}_{t}$, i.e., $\boldsymbol{u}_{t}=u_{t} \boldsymbol{s}, u_{p}=v_{p}+u_{n}$, and the change in position vector due to increments in the intrinsic coordinates with $t$ fixed is $d \boldsymbol{x}=a(1+n \kappa) d \xi \boldsymbol{s}+d n \boldsymbol{n}$. When the incompressibility condition is evaluated in the limit as $n \rightarrow 0$, the first term tends to the surface divergence $\nabla_{s} \cdot \boldsymbol{u}_{s}$, and since the normal speed $u_{n}$ of the surface is independent of $n$, so that $\partial_{n} u_{p}=\partial_{n} v_{p}$, the condition implies that

$$
\left.\frac{\partial v_{p}}{\partial n}\right|_{\partial \Omega}=-\kappa u_{n}-\nabla_{s} \cdot \boldsymbol{u}_{s}
$$

where the right-hand side contains surface data alone. Both (2.14) and (2.15) hold in three as well as two dimensions, while (2.16) is generalized to three dimensions by replacing $\kappa$ with the sum of the principal curvatures, $\kappa_{1}+\kappa_{2}$.

The transfer coefficient $J$ in the bulk-interface surfactant exchange term $\left.J \partial_{n} C\right|_{\partial \Omega}$ of (2.7) is rescaled by putting $J=\epsilon J_{0}$, where $J_{0}=O(1)$, so that the exchange term remains $O(1)$ when expressed in terms of the rescaled coordinate $N$. The equation becomes

$$
\left.\frac{\partial \Gamma}{\partial t}\right|_{\xi}-\left.\frac{\partial \boldsymbol{X}}{\partial t}\right|_{\xi} \cdot \nabla_{s} \Gamma+\nabla_{s} \cdot\left(\Gamma \boldsymbol{u}_{s}\right)+\Gamma \kappa u_{n}=\frac{1}{P e_{s}} \nabla_{s}^{2} \Gamma+\left.J_{0} \frac{\partial C}{\partial N}\right|_{\partial \Omega}, \quad \boldsymbol{x} \in \partial \Omega .
$$


The initial boundary value problem (2.14) and (2.15) for $C$ within the transition layer and the rescaled bulk-interface exchange term of (2.17) have been constructed by a leading order singular perturbation rescaling of the full equations in the limit $P e \rightarrow \infty$. As a result, the expansion parameter $P e$ does not appear in the rescaled model.

The bulk and surface surfactant diffusivities, $D$ and $D_{s}$, are believed to be of the same order of magnitude [23], so that the respective Péclet numbers are too. However, the surface diffusion of adsorbed surfactant, which is represented by the term $P e_{s}^{-1} \nabla_{s}^{2} \Gamma$ in (2.17), is not given the same asymptotic treatment. One reason for this is that near-discontinuities in the surface concentration $\Gamma$ have not been found in the computations of this study, although we see numerically large changes in the first derivative of $\Gamma$ near accumulation points of surfactant in the examples of section 6 . We note that large gradients of surface surfactant concentration have been observed in a number of studies for which capillary-induced pinch-off occurs; see, for example, $[25,26,27]$. However, since the dimensionality of a near-discontinuity in $\Gamma$ on an interface is one lower than that of a near-discontinuity in the bulk concentration $C$, it is less computationally expensive to resolve it by decreasing the mesh size. Although the influence of surface diffusion can be included in the analysis and computations, its influence on the results of this study is sufficiently small that it is neglected.

3. The surface-based Stokes flow solver. In principle, any surface-based method that can be used to solve examples of Stokes flow in the presence of insoluble surfactant can be adapted to solve examples that include surfactant solubility effects in the infinite Péclet number limit, as outlined in section 2.1.

Here we use the Sherman-Lauricella formulation of the boundary integral method. This is an indirect method, for which the primitive variables are expressed in terms of a single complex density $\omega$ that is defined on the drop interface and satisfies a Fredholm second kind integral equation. It has been used to solve the biharmonic equation in two dimensions for problems of static elasticity (see, for example, [20, 21]) and more recently to solve Stokes equations for fluid flow (see [28, 29, 30, 31]).

In terms of Cartesian components, the velocity of the exterior flow is $\boldsymbol{u}=\left(u_{1}, u_{2}\right)$ and the velocity of the interior flow is $\boldsymbol{u}^{i}=\left(u_{1}^{i}, u_{2}^{i}\right)$. A stream function is introduced for each region, so that

$$
\left(u_{1}, u_{2}\right)=\left(W_{x_{2}},-W_{x_{1}}\right), \quad \boldsymbol{x} \in \Omega,
$$

and $\left(u_{1}^{i}, u_{2}^{i}\right)=\left(W_{x_{2}}^{i},-W_{x_{1}}^{i}\right)$ for $\boldsymbol{x} \in \Omega^{i}$. Since the formulation in both regions is similar, we focus on the exterior region $\Omega$ and give only the analogous results for the interior region as needed. The curl of (2.1a) implies that

$$
\nabla^{4} W=0, \quad x \in \Omega
$$

that is, $W\left(x_{1}, x_{2}\right)$ is a biharmonic function. Similarly $W^{i}\left(x_{1}, x_{2}\right)$ is biharmonic on $\Omega^{i}$. The stream function $W\left(x_{1}, x_{2}\right)$ therefore has a Goursat representation,

$$
W\left(x_{1}, x_{2}\right)=\operatorname{Re}(\bar{z} f(z)+h(z)), \quad z \in \Omega,
$$

where $f(z)$ and $h(z)$ are analytic functions of the complex variable $z=x_{1}+i x_{2}$ on $\Omega[21,32]$. The functions $f(z)$ and $g(z)=h^{\prime}(z)$ are known as Goursat functions. Similarly, $W^{i}\left(x_{1}, x_{2}\right)=\operatorname{Re}\left(\bar{z} f^{i}(z)+h^{i}(z)\right)$ for $z \in \Omega^{i}$, where $f^{i}(z)$ and $h^{i}(z)$, with $g^{i}(z)=h^{i \prime}(z)$, are analytic in $\Omega^{i}$. 
The primitive variables and their spatial derivatives in the exterior and interior regions can be expressed in terms of the Goursat functions; see, for example, [33]. In the exterior domain, $z \in \Omega$,

$$
\begin{aligned}
-u_{2}+i u_{1} & =f(z)+z \overline{f^{\prime}(z)}+\overline{g(z)}, \\
q+i p & =-4 f^{\prime}(z), \\
e_{11}+i e_{12} & =-e_{22}+i e_{21}=-i\left(z \overline{f^{\prime \prime}(z)}+\overline{g^{\prime}(z)}\right) .
\end{aligned}
$$

Here, $q$ is the fluid vorticity, with $\boldsymbol{\omega}=\nabla \times \boldsymbol{u}=\left(\partial_{x_{1}} u_{2}-\partial_{x_{2}} u_{1}\right) \boldsymbol{e}_{3}=q \boldsymbol{e}_{3}$. Analogous expressions hold for the interior domain, with the exception that $q^{i}+i\left(p^{i} / \lambda\right)=$ $-4 f^{i \prime}(z)$ for $z \in \Omega^{i}$.

The left-hand side of (3.1a) and (3.1b) can be evaluated as $|z| \rightarrow \infty$ from the imposed far-field flow (2.11), which implies that with no mass source in the drop interior the vorticity $q=G+O\left(|z|^{-3}\right)$ and the pressure $p=p_{\infty}+O\left(|z|^{-3}\right)$. The ambient pressure $p_{\infty}$ can be set to zero without loss of generality, and then

$$
\begin{aligned}
& f(z)=\frac{G}{4} z+H(t)+O\left(|z|^{-2}\right), \\
& g(z)=-(B+i Q) z-\overline{H(t)}+O\left(|z|^{-2}\right),
\end{aligned}
$$

as $|z| \rightarrow \infty$, where $H(t)$ is an as yet arbitrary function of time.

The surface stress exerted on the interface $\partial \Omega$ by material in the exterior domain, per (2.4), is $-p \boldsymbol{n}+2 \boldsymbol{e} \cdot \boldsymbol{n}=\left(f_{1}, f_{2}\right)$, which has complex counterpart

$$
\left.f_{1}+i f_{2}=2 \frac{\partial}{\partial s}\left\{\lim _{z \rightarrow \tau^{+}}\left(f(z)-z \overline{f^{\prime}(z)}\right)-\overline{g(z)}\right)\right\},
$$

where the limit indicates that $z$ approaches a point $\tau$ on $\partial \Omega$ from the exterior domain. A similar expression multiplied by $\lambda$ holds for the surface stress due to material in the interior domain. The difference is equal to the interfacial stress due to surface tension, which is given by the right-hand side of (2.4) and has complex counterpart $-\partial_{s}\left(\sigma \partial_{s} \tau\right)$. All terms in the stress-balance boundary condition are seen to be perfect derivatives with respect to $s$, and freedom of choice in specifying the Goursat functions allows us to set to zero a function of time that results in integrating the boundary condition with respect to $s$. The result is that the stress-balance boundary condition takes the form

$$
\lim _{z \rightarrow \tau^{+}}\left(f(z)-z \overline{f^{\prime}(z)}-\overline{g(z)}\right)-\lambda \lim _{z \rightarrow \tau^{-}}\left(f^{i}(z)-z \overline{f^{i \prime}(z)}-\overline{g^{i}(z)}\right)=-\frac{\sigma \tau_{s}}{2} .
$$

In the Sherman-Lauricella formulation, the Goursat functions are written in terms of Cauchy-type integrals that contain a single complex density $\omega(z, t)$ defined on the time-evolving interface $\partial \Omega$, and where the integrals give the modification to the imposed far-field flow that is caused by the drop. The representation is such that, if we introduce

$$
\begin{aligned}
f^{o}(z)= & \frac{1}{2 \pi i} \int_{\partial \Omega} \frac{\omega(\zeta, t)}{\zeta-z} d \zeta+\frac{G z}{4}+H(t) \\
g^{o}(z)= & \frac{1}{2 \pi i} \int_{\partial \Omega} \frac{-\overline{\omega(\zeta, t)} d \zeta+\omega(\zeta, t) d \bar{\zeta}}{\zeta-z}-\frac{1}{2 \pi i} \int_{\partial \Omega} \frac{\bar{\zeta} \omega(\zeta, t)}{(\zeta-z)^{2}} d \zeta \\
& -(B+i Q) z-\overline{H(t)}
\end{aligned}
$$

Copyright $@$ by SIAM. Unauthorized reproduction of this article is prohibited. 
then the Goursat functions are given by

$$
\begin{aligned}
(f(z), g(z)) & =\left(f^{o}(z), g^{o}(z)\right) & & \text { when } z \in \Omega, \\
\left(f^{i}(z), g^{i}(z)\right) & =\left(f^{o}(z), g^{o}(z)\right) & & \text { when } z \in \Omega^{i} .
\end{aligned}
$$

Here, $z$ is an arbitrary point in the complex plane away from the interface $\partial \Omega$, and $\zeta$ is the variable of integration on the interface contour. In the definitions (3.3a) and $(3.3 \mathrm{~b}), \int_{\partial \Omega}$ can denote integration around $\partial \Omega$ in either the counterclockwise direction, as is the usual convention in the complex plane, or clockwise; the difference is resolved by a change in sign of $\omega(\zeta, t)$, and we choose the clockwise direction. The time dependence of $\omega(\zeta, t)$ and $H(t)$ is indicated explicitly, while the time dependence of the Goursat functions $f(z)$ and $g(z)$ is taken to be understood. Time enters only via the time derivative in the kinematic condition (2.3) and in the equations for bulk and surface concentrations of surfactant (2.14) and (2.17), and, as may be chosen, parametrically via the parameters $Q, B$, and $G$ of the imposed flow (2.11).

From the definitions (3.3a) and (3.3b), the Goursat functions are analytic functions of $z$ except for $z$ on the contour $\partial \Omega$. They are also singular as $z \rightarrow \infty$ to accommodate the imposed flow. The Sherman-Lauricella integral equation is constructed when the representation of the Goursat functions in terms of $\omega$ of (3.3a) to (3.4b) is substituted into the stress-balance boundary condition (3.2). As $z$ approaches a point $\tau$ on $\partial \Omega$, some of the Cauchy-type integrals that result have local, simple pole contributions from a neighborhood of $z=\tau$ that can be evaluated by the Plemelj formulas [32], and the remaining part of these integrals is of principal value type. The final form that the equation takes can be written as

$$
\begin{aligned}
\omega(\tau, t)+ & \frac{\beta}{2 \pi i} \int_{\partial \Omega} \omega(\zeta, t) d \ln \frac{\zeta-\tau}{\bar{\zeta}-\bar{\tau}}+\frac{\beta}{2 \pi i} \int_{\partial \Omega} \overline{\omega(\zeta, t)} d \frac{\zeta-\tau}{\bar{\zeta}-\bar{\tau}} \\
& +\beta(B-i Q) \bar{\tau}+2 \beta H(t)=-\frac{\gamma}{2} \sigma(\Gamma) \frac{\partial \tau}{\partial s},
\end{aligned}
$$

where $\beta=\frac{1-\lambda}{1+\lambda}, \gamma=\frac{1}{1+\lambda}$, and it turns out that the apparent singularity at $\zeta=\tau$ in the two integrals on the left-hand side is removable. The choice that

$$
H(t)=\frac{1}{2} \int_{\partial \Omega} \omega(\zeta, t) d s
$$

removes a rank deficiency of the integral equation (3.5) in the limit when $\lambda=0$ of an inviscid drop (see, for example, $[20,29]$ ), and $H(t) \equiv 0$ as a consequence of the constant area of the interior region $\Omega^{i}$.

The fluid velocity on the interface is found from (3.1a) by letting $z$ approach a point $\tau$ on $\partial \Omega$ from either $\Omega$ or $\Omega^{i}$. The representation of the Goursat functions in terms of $\omega(z, t)$ of (3.3a) to (3.4b) is such that the local, simple pole contributions to (3.1a) from the integrals near $z=\tau$ cancel as $z \rightarrow \tau^{ \pm}$. The fluid velocity is therefore continuous automatically across the interface and is given by

$$
\begin{aligned}
u_{1}+\left.i u_{2}\right|_{\partial \Omega}=- & \frac{1}{2 \pi} f_{\partial \Omega} \omega(\zeta, t)\left(\frac{d \zeta}{\zeta-\tau}+\frac{d \bar{\zeta}}{\bar{\zeta}-\bar{\tau}}\right) \\
& +\frac{1}{2 \pi} \int_{\partial \Omega} \overline{\omega(\zeta, t)} d\left(\frac{\zeta-\tau}{\bar{\zeta}-\bar{\tau}}\right)+(Q+i B) \bar{\tau}-\frac{i G}{2} \tau
\end{aligned}
$$

on the interface. The apparent singularity for $\zeta$ near $\tau$ in the second integral is removable, but in the first integral the bar indicates that it is to be interpreted as a Cauchy principal value integral. 
The fluid velocity on the interface, in terms of its normal and tangential components $u_{n}$ and $u_{s}$, is $\boldsymbol{u}=u_{n} \boldsymbol{n}+u_{s} \boldsymbol{s}$, where the complex counterparts of the unit vectors $\boldsymbol{n}$ and $s$ are $n$ and $s_{T}$ with $s_{T}=-i n=\partial_{s} \tau$. It follows that

$$
u_{n}=\Re\left\{\left.\left(u_{1}+i u_{2}\right)\right|_{\partial \Omega} \bar{n}\right\} \text { and } u_{s}=-\Im\left\{\left.\left(u_{1}+i u_{2}\right)\right|_{\partial \Omega} \bar{n}\right\}
$$

on the interface.

For the numerical discretization of (3.5) given below, two different parameterizations of the interface $\partial \Omega$ are introduced that have different distributions of the computational mesh points. Both are constructed using an approach due to Hou, Lowengrub, and Shelley [34], and we include some details here for completeness.

The spatial parameterization of the interface is given by $\alpha \in[0,2 \pi)$, and a point $\tau$ on the interface has Cartesian coordinates $\left(x_{1}, x_{2}\right)$, so that $\tau=x_{1}(\alpha, t)+i x_{2}(\alpha, t)$. The unit tangent vector $s_{T}$ and normal $n$ in complex form are $s_{T}=\frac{\partial \tau}{\partial s}=\tau_{\alpha} / s_{\alpha}=e^{i \theta}$ and $n=i s_{T}=i e^{i \theta}$, where $s_{\alpha}=\left|\tau_{\alpha}\right|$. Differentiation with respect to time implies that $\tau_{\alpha t}=s_{\alpha t} e^{i \theta}+s_{\alpha} \theta_{t} i e^{i \theta}$.

When $\tau$ is a material point on the interface its velocity is equal to the local fluid velocity, per (2.2), so that

$$
\frac{d \tau}{d t}=u_{n} i e^{i \theta}+u_{s} e^{i \theta}
$$

However, the shape of the evolving interface is determined by the normal velocity component $u_{n}$ alone. Although $u_{s}$ has physical meaning as the tangential component of the fluid velocity, if $u_{s}$ is replaced by any other smooth function $\phi_{s}(\alpha, t)$ in (3.8), then $\tau$ still lies on the interface but is no longer a material point, and the role of $\phi_{s}$ is simply to implement a specific choice of $\tau \in \partial \Omega$ and the interface parameterization via $\alpha$, without changing the interface shape or evolution.

When this is done, differentiation of (3.8) with respect to $\alpha$ gives a second relation for $\tau_{\alpha t}$,

$$
\tau_{\alpha t}=\left(\left(\phi_{s}\right)_{\alpha}-u_{n} \theta_{\alpha}\right) e^{i \theta}+\left(\left(u_{n}\right)_{\alpha}+\phi_{s} \theta_{\alpha}\right) i e^{i \theta} .
$$

Equating these two expressions for $\tau_{\alpha t}$, we have

$$
\begin{aligned}
s_{\alpha t} & =\left(\phi_{s}\right)_{\alpha}-u_{n} \theta_{\alpha}, \\
\theta_{t} & =\frac{1}{s_{\alpha}}\left(\left(u_{n}\right)_{\alpha}+\phi_{s} \theta_{\alpha}\right),
\end{aligned}
$$

where $\partial \Omega$ is now described parametrically by $s=s(\alpha, t)$ and $\theta=\theta(\alpha, t)$ instead of $x_{1}=x_{1}(\alpha, t)$ and $x_{2}=x_{2}(\alpha, t)$.

The quadratures in (3.5) are evaluated by the trapezoidal rule with a fixed step size in $\alpha \in[0,2 \pi)$, which gives spectral accuracy. The Cauchy principal value integral in (3.6) is computed by the Van de Vooren correction [35, 36]. The two parameterizations chosen for the interface produce (i) an equal arc length frame, which is used for the mesh-based computation of the transition layer described in section 4, and (ii) a Lagrangian frame, which is used for the Green's function solution of the transition layer of section 5 .

The first of these is determined by setting $s_{\alpha}$ to be constant along the interface, although it varies in time. Then, since $s_{\alpha}$ is always equal to its mean or average around $\partial \Omega$, it follows from (3.10a) that

$$
s_{\alpha t}=\left(\phi_{s}\right)_{\alpha}-u_{n} \theta_{\alpha}=-\frac{1}{2 \pi} \int_{0}^{2 \pi} u_{n} \theta_{\alpha^{\prime}} d \alpha^{\prime} .
$$

Copyright (C) by SIAM. Unauthorized reproduction of this article is prohibited. 
Integration of the second of these equations with respect to $\alpha$ implies that

$$
\phi_{s}(\alpha, t)=-\frac{\alpha}{2 \pi} \int_{0}^{2 \pi} u_{n} \theta_{\alpha^{\prime}} d \alpha^{\prime}+\int_{0}^{\alpha} u_{n} \theta_{\alpha^{\prime}} d \alpha^{\prime}
$$

where an arbitrary function of time has been set to zero. This gives the required tangential velocity $\phi_{s}$ of the equal arc length frame.

When (3.12) is substituted into (3.10a) and (3.10b), the system by which the dynamics of the interface is tracked becomes

$$
\begin{gathered}
s_{\alpha t}=-\frac{1}{2 \pi} \int_{0}^{2 \pi} u_{n} \theta_{\alpha^{\prime}} d \alpha^{\prime} \\
\theta_{t}=\frac{1}{s_{\alpha}}\left[\theta_{\alpha}\left(\int_{0}^{\alpha} u_{n} \theta_{\alpha^{\prime}} d \alpha^{\prime}-\frac{\alpha}{2 \pi} \int_{0}^{2 \pi} u_{n} \theta_{\alpha^{\prime}} d \alpha^{\prime}\right)+\left(u_{n}\right)_{\alpha}\right] .
\end{gathered}
$$

At each time step (3.13a) and (3.13b) are integrated forward in time, and $\left(s_{\alpha}, \theta\right)$ are mapped to the Cartesian coordinates $\left(x_{1}, x_{2}\right)$ of points on $\partial \Omega$. The map is given by integration of $\tau_{\alpha}=s_{\alpha} e^{i \theta}$ with respect to $\alpha$ and is

$$
\begin{aligned}
& x_{1}(\alpha, t)=x_{1}(0, t)+s_{\alpha}(t) \int_{0}^{\alpha} \cos \left(\theta\left(\alpha^{\prime}, t\right)\right) d \alpha^{\prime}, \\
& x_{2}(\alpha, t)=x_{2}(0, t)+s_{\alpha}(t) \int_{0}^{\alpha} \sin \left(\theta\left(\alpha^{\prime}, t\right)\right) d \alpha^{\prime},
\end{aligned}
$$

where $\left(x_{1}(0, t), x_{2}(0, t)\right)$ is the position of the point $\alpha=0$ at time $t$, which is evolved from the kinematic condition and is hence given by $(3.8)$ with $u_{s}(0, t)=0$.

In the second case, (ii), of a Lagrangian frame, $\phi_{s}=u_{s}$ is the surface tangential fluid velocity, and $\alpha$ is the initial arc length $s_{0}$ of a material particle along the interface relative to a chosen origin $O^{\prime}$, which lies in $[0,2 \pi)$ since the dimensionless initial drop radius is 1 .

4. A mesh-based solution for the transition layer. The transition layer, where the advection-diffusion equation (2.14) holds, is defined on a rectangular domain. For the mesh-based method, $\alpha \in[0,2 \pi)$ is a normalized equal arc length parameter for the interface $\partial \Omega$, as introduced in (3.11) and (3.12). The bulk surfactant concentration in the transition layer $C(\alpha, N, t)$ is $2 \pi$-periodic in $\alpha$ and satisfies initial and boundary conditions (2.15). The problem for $C(\alpha, N, t)$ is therefore

$$
\begin{aligned}
\frac{\partial C}{\partial t}+v_{s}(\alpha, t) \frac{\partial C}{\partial s}+\psi(\alpha, t) N \frac{\partial C}{\partial N} & =\frac{\partial^{2} C}{\partial N^{2}} \\
\text { where } v_{s}(\alpha, t)=u_{s}(\alpha, t)-U_{s}(\alpha, t) \text { and } \psi(\alpha, t) & =-\left(\kappa u_{n}+\frac{\partial u_{s}}{\partial s}\right), \\
\text { with initial condition } C(\alpha, N, t=0) & =1,
\end{aligned}
$$
and boundary conditions $C(0, N, t)=C(2 \pi, N, t)$, with

$$
C(\alpha, N=0, t)=\frac{\Gamma(\alpha, t)}{K(1-\Gamma(\alpha, t))}, \quad C(\alpha, N, t) \rightarrow 1 \quad \text { as } N \rightarrow \infty .
$$

The mesh-based method is spectrally accurate in space and second order in time. Chebyshev-Lobatto collocation points are introduced in the normal $N$ direction with

$$
N_{j}=\frac{N_{f}}{2}\left(\cos \left(\frac{j \pi}{m}\right)+1\right), \quad j=0,1, \ldots, m,
$$


which cluster near the interface $N=0$, and a finite far-field boundary location $N=$ $N_{f}$, at which the condition $C=1$ is imposed. The computations are usually found to be well resolved when $N_{f}$ is set to 20. The second derivative $\partial_{N}^{2} C$ of (4.1a) is calculated implicitly via a Chebyshev differentiation matrix [37]. The first spatial derivative terms of (4.1a) as well as the spatial derivatives in the surface surfactant equation (2.17) are found explicitly via FFTs. The time step uses a semi-implicit, two-step variation of the Crank-Nicholson method, which is solved by GMRES.

5. A Green's function solution for the transition layer. When the formulation for bulk-interface surfactant exchange is time-dependent but spatially $1 \mathrm{D}$, with no flow and a planar interface with no variation in the tangential direction, Ward and Tordai [38] have expressed the solution for the surface surfactant concentration in terms of an integral equation over time that relates the surface concentration $\Gamma$ to the bulk concentration $\left.C\right|_{\partial \Omega}$ neighboring the interface. In the diffusion-controlled limit of (2.9), this gives a closed, well-posed problem for the surface concentration $\Gamma$ as a function of time $t$. A review of this is given in [23], and a more recent study is given in [39].

In this section, we take a similar approach but in a more general context. We consider a nonplanar interface, with flow in the large bulk Péclet number limit, so that the length scale for quantities such as the fluid velocity and interface curvature is much greater than the width of the bulk-interface surfactant exchange layer. The dependent variables are also allowed to vary in the direction tangential to the interface.

In terms of the interface arc length coordinate $s$, the problem (4.1a)-(4.1c) is

$$
\begin{gathered}
\frac{\partial C}{\partial t}+v_{s}(s, t) \frac{\partial C}{\partial s}+\psi(s, t) N \frac{\partial C}{\partial N}=\frac{\partial^{2} C}{\partial N^{2}}, \\
\text { where } v_{s}(s, t)=u_{s}(s, t)-U_{s}(s, t) \text { and } \psi(s, t)=-\left(\kappa u_{n}+\frac{\partial u_{s}}{\partial s}\right), \\
\text { with initial condition } C(s, N, t=0)=1, \\
\quad \text { and boundary conditions } \\
C(s, N=0, t)=\frac{\Gamma(s, t)}{K(1-\Gamma(s, t))}, C(s, N, t) \rightarrow 1 \quad \text { as } N \rightarrow \infty,
\end{gathered}
$$

with periodicity of $C$ in $s$.

It is useful to introduce characteristic paths

$$
s=f\left(s_{0}, t\right) \quad \text { such that } \frac{\partial s}{\partial t}=v_{s}(s, t), \text { with } s=s_{0} \text { at } t=0 .
$$

Since $v_{s}(s, t)$ is the tangential fluid velocity on the interface in the intrinsic frame, the characteristic coordinate $s_{0}$ is a label for material particle paths, or a Lagrangian coordinate, on the interface. A change of variables from the frame $(s, N, t)$ to the frame $\left(s_{0}, N, t\right)$ given by (5.2) with $N$ and $t$ unchanged absorbs the term $v_{s} \partial_{s} C$ of (5.1a) into the time derivative in the new frame, so that $s_{0}$ appears only as a parameter. If we introduce a shift in $C$,

$$
\tilde{C}=C-1,
$$

then drop the tilde and suppress the dependence on $s_{0}$ by defining

$$
\begin{gathered}
\psi_{0}(t)=\psi\left(s=f\left(s_{0}, t\right), t\right), \quad \Gamma_{0}(t)=\Gamma\left(s=f\left(s_{0}, t\right), t\right), \\
\text { and } h_{0}(t)=\frac{\Gamma_{0}(t)}{K\left(1-\Gamma_{0}(t)\right)}-1,
\end{gathered}
$$


the problem becomes

$$
\frac{\partial C}{\partial t}+\psi_{0}(t) N \frac{\partial C}{\partial N}=\frac{\partial^{2} C}{\partial N^{2}},
$$

with initial condition $C(N, t=0)=0$,

(5.5c) and boundary conditions $C(N=0, t)=h_{0}(t), \quad C(N \rightarrow \infty, t)=0$.

This can be solved by Duhamel's principle [40]. If $u(N, t, \tau)$ is the solution of (5.5a) with step function boundary data, so that

$$
\begin{aligned}
\frac{\partial u}{\partial t}+\psi_{0}(t) & N \frac{\partial u}{\partial N}=\frac{\partial^{2} u}{\partial N^{2}}, \\
\text { with } u(N, t, \tau) & =0 \text { for } 0 \leq t \leq \tau \quad(\tau \text { arbitrary }), \\
\text { and } u(N=0, t, \tau)=1 \text { for } 0 & \leq \tau<t, \quad u(N \rightarrow \infty, t, \tau)=0,
\end{aligned}
$$

then the solution of $(5.5 \mathrm{a})-(5.5 \mathrm{c})$ is

$$
C(N, t)=-\int_{0}^{t} \frac{\partial u}{\partial \tau}(N, t, \tau) h_{0}(\tau) d \tau .
$$

To solve (5.6a)-(5.6c) we look for an error function-like solution and introduce a similarity variable

$$
\eta(N, t)=\frac{N}{\gamma(t)}
$$

where $\gamma(t)$ is a function of time that is to be found. Although the data (5.6b)-(5.6c) for $u$ is time-invariant, i.e., it does not change under a translation in $t$, the PDE (5.6a) is not time-invariant because of the time dependence of $\psi_{0}(t)$. As a consequence, $u$ and $\gamma$ are functions of $t$ and $\tau$ separately, not solely via the combination $t-\tau$. They also depend parametrically on $s_{0}$, via the dependence of $\psi_{0}(t)$ on $s_{0}$. The derivatives with respect to $t$ and $N$ transform as $\partial_{t} \mapsto \partial_{t}-(\eta / \gamma) \gamma_{t} \partial_{\eta}$ and $\partial_{N} \mapsto(1 / \gamma) \partial_{\eta}$, and we look for a solution that depends on $t$ through $\gamma$ alone, so that after some rearrangement (5.6a) becomes

$$
\psi_{0}(t) \gamma^{2}-\gamma \gamma_{t}=\frac{u_{\eta \eta}}{\eta u_{\eta}}=\Theta
$$

Here, since $\eta$ and $t$ are independent variables, $\Theta$ is a constant. Its value can be chosen arbitrarily, as is seen by noting that $\Theta$ is scaled out of (5.9) by setting $\eta^{\prime}=\Theta^{\frac{1}{2}} \eta$ and $\gamma^{\prime}=\Theta^{-\frac{1}{2}} \gamma$, with $\eta^{\prime}=N / \gamma^{\prime}$. To simplify the calculation, we set $\Theta=-2$, so that

$$
\begin{aligned}
\gamma \gamma_{t}-\psi_{0}(t) \gamma^{2} & =2, \\
u_{\eta \eta}+2 \eta u_{\eta} & =0
\end{aligned}
$$

We anticipate an error function-like solution for $u$, where $\gamma=0$ for $t \in[0, \tau]$ and $\gamma \rightarrow 0^{+}$as $t \rightarrow \tau^{+}$. From (5.10a), we find that

$$
\gamma^{2}(t-\tau, \tau)=4 \int_{0}^{t-\tau} e^{2 \int_{\tilde{t}}^{t-\tau} \psi_{0}\left(t^{\prime}+\tau\right) d t^{\prime}} d \tilde{t}
$$

which has the required behavior as $t \rightarrow \tau^{+}$. In terms of the function $a(t, \tau)$ defined by

$$
a(t, \tau)=2 \int_{0}^{t} \psi_{0}\left(t^{\prime}+\tau\right) d t^{\prime}
$$

Copyright $@$ by SIAM. Unauthorized reproduction of this article is prohibited. 
the exponent in (5.11) is $a(t-\tau, \tau)-a(\tilde{t}, \tau)$, and the solution for $\gamma$ can be written

$$
\gamma(t-\tau, \tau)=2 e^{\frac{1}{2} a(t-\tau, \tau)}\left(\int_{0}^{t-\tau} e^{-a(\tilde{t}, \tau)} d \tilde{t}\right)^{1 / 2} .
$$

We note that the argument of $\psi_{0}$ in the solution (5.11) for $\gamma$ and in the definition (5.12) of $a$ is advanced by $\tau$. This is a consequence of the step function boundary data of (5.6b) and (5.6c) being zero for $t \in[0, \tau]$ and $u=1$ for $t>\tau$, at which time $\psi_{0}(t)=\psi_{0}(\tau)$. In evaluating $\psi_{0}(t)$, we also note that during the interval $t \in[0, \tau]$ the original arc length coordinate $s=f\left(s_{0}, t\right)$ advances from its initial position $s=s_{0}=$ $f\left(s_{0}, 0\right)$ at time $t=0$ to position $s=f\left(s_{0}, \tau\right)$ before the boundary data switches to $u=1$ for $t>\tau$. This is seen in the definition for $\psi_{0}(t)=\psi\left(s=f\left(s_{0}, t\right), t\right)$ of $(5.4 \mathrm{a})$.

We now turn to (5.10b). The boundary conditions (5.6c) imply that for $t>\tau$ the solution is

$$
u(\eta)=1-\frac{2}{\sqrt{\pi}} \int_{0}^{\eta} e^{-\tilde{\eta}^{2}} d \tilde{\eta},
$$

where $\operatorname{erf}(\eta)=-\frac{2}{\sqrt{\pi}} \int_{0}^{\eta} e^{-\tilde{\eta}^{2}} d \tilde{\eta}$ is the error function. The solution to the problem (5.6a) $-(5.6 \mathrm{c})$ with step function boundary condition is therefore

$$
u(N, t, \tau)=1-\frac{2}{\sqrt{\pi}} \int_{0}^{\frac{N}{\gamma}} e^{-\tilde{\eta}^{2}} d \tilde{\eta}, \quad \text { where } \gamma=\gamma(t-\tau, \tau),
$$

the similarity variable $\eta=N / \gamma$ of (5.8) has been substituted, and $\gamma=\gamma(t-\tau, \tau)$ is given by (5.12) and (5.13).

Duhamel's principle (5.7) can now be applied, and it gives the solution for $C$ as a convolution integral in time. The time derivative $\partial_{\tau} u(N, t, \tau)$ is found from (5.15); then, changing the variable of integration in the integral from $\tau$ to $u=t-\tau$ and recalling (5.3), we have the solution of the problem (5.1a)-(5.1c) for $C$ in the $\left(s_{0}, N, t\right)$ frame in the form

$$
C\left(s_{0}, N, t\right)=\frac{2 N}{\sqrt{\pi}} \int_{0}^{t} e^{-\left(\frac{N}{\gamma}\right)^{2}} \frac{\partial_{u} \gamma}{\gamma^{2}} h_{0}(t-u) d u+1,
$$

where $\gamma=\gamma(u, t-u)$. The parametric dependence of this solution on $s_{0}$ appears in the dependence of $\psi_{0}(t)$ and $h_{0}(t)$ on $s_{0}$, as defined by (5.4a) and (5.4b), and in the dependence of $a(t, \tau)$ and $\gamma(t-\tau, \tau)$ on $\psi_{0}(t)$, as given by (5.12) and (5.13). In the limit when $\psi_{0}(t)=0$, the problem simplifies to the heat equation, and the expression for $\gamma$ recovers the familiar result that $\gamma(u, t-u)=2 \sqrt{u}$.

The result (5.16) can be used to find the normal derivative $\left.\partial_{N} C\right|_{N=0}$ at the interface and to evaluate the bulk-interface surfactant exchange term of (2.7) and (2.17). However, the limit $N \rightarrow 0$ needs to be formulated with some care. To resolve this, we decompose the integral in (5.16) into two parts, over $u \in[0, \delta]$ and $u \in[\delta, t]$ for some small $\delta>0$, and treat each part separately. Before doing so, we first need the behavior of $\gamma(u, t-u)$ as $u \rightarrow 0$.

To find this, we note from (5.13) that

$$
\gamma(u, t-u)=2 e^{\frac{1}{2} a(u, t-u)}\left(\int_{0}^{u} e^{-a(\tilde{t}, t-u)} d \tilde{t}\right)^{\frac{1}{2}},
$$

Copyright $@$ by SIAM. Unauthorized reproduction of this article is prohibited. 
so that from the definition of $a$ at (5.12), for small $u$,

$$
a(u, t-u)=2 \int_{0}^{u} \psi_{0}\left(t^{\prime}+t-u\right) d t^{\prime}=2 \psi_{0}(t) u+O\left(u^{2}\right)
$$

as $u \rightarrow 0$, with a similar result for $a(\tilde{t}, t-u)$. From these last results it follows that as $u \rightarrow 0$

$$
\begin{aligned}
\gamma(u, t-u) & =2 \sqrt{u}\left(1+\frac{\psi_{0}(t)}{2} u+O\left(u^{2}\right)\right), \\
\partial_{u} \gamma(u, t-u) & =u^{-1 / 2}\left(1+\frac{3 \psi_{0}(t)}{2} u+O\left(u^{2}\right)\right) .
\end{aligned}
$$

First, consider the contribution $C_{1}$ to the integral for $C$ in (5.16) when $u \in[0, \delta]$, where $\delta$ is arbitrary but small and positive and $N \geq 0$. From (5.19), expansion of the integrand, if $h_{0}$ is sufficiently smooth to have a Taylor expansion about $u=0$, implies that

$$
\begin{aligned}
C_{1}(N, t) & =\frac{2 N h_{0}(t)}{\sqrt{\pi}} e^{\frac{\psi_{0}(t) N^{2}}{4}} \int_{0}^{\delta} \frac{e^{-\frac{N^{2}}{4 u}}}{4 u^{\frac{3}{2}}}(1+O(u)) d u \\
& =\frac{2 h_{0}(t)}{\sqrt{\pi}} e^{\frac{\psi_{0}(t) N^{2}}{4}} \int_{\frac{N}{2 \sqrt{\delta}}}^{\infty} e^{-s^{2}}\left(1+O\left(\frac{N^{2}}{s^{2}}\right)\right) d s,
\end{aligned}
$$

where $s=N / 2 \sqrt{u}$. Note that if we let $N \rightarrow 0$ in this last result, the integral tends to $\sqrt{\pi} / 2$, and $C_{1}$ approaches $h_{0}(t)$, so that $C_{1}$ recovers the boundary data of (5.3) and (5.5c), which is $C\left(s_{0}, N=0, t\right)=1+h_{0}(t)$. Returning to the derivative $\partial_{N} C_{1}$, we find that the contribution from $u \in[0, \delta]$ is

$$
\left.\frac{\partial C_{1}}{\partial N}\right|_{N=0}=-\frac{h_{0}(t)}{\sqrt{\pi \delta}}+O\left(\delta^{\frac{1}{2}}\right) .
$$

Second, to evaluate the contribution $C_{2}$ to the integral of (5.16) when $u \in[\delta, t]$, we note that since $u$ is bounded away from zero, $\gamma(u, t-u)$ is bounded away from zero, and the derivative with respect to $N$ can be taken directly. Then, letting $N \rightarrow 0$ and putting $\gamma^{-2} \partial_{u} \gamma=-\partial_{u}\left(\gamma^{-1}\right)$, we have

$$
\left.\frac{\partial C_{2}}{\partial N}\right|_{N=0}=-\frac{2}{\sqrt{\pi}} \int_{\delta}^{t} \frac{\partial}{\partial u}\left(\frac{1}{\gamma(u, t-u)}\right) h_{0}(t-u) d u .
$$

An integration by parts with the relation (5.19) for $\gamma(\delta, t-\delta)$ gives the contribution

$$
\left.\frac{\partial C_{2}}{\partial N}\right|_{N=0}=\frac{h_{0}(t)}{\sqrt{\pi \delta}}+O\left(\delta^{\frac{1}{2}}\right)-\frac{2 h_{0}(0)}{\sqrt{\pi} \gamma(t, 0)}+\frac{2}{\sqrt{\pi}} \int_{\delta}^{t} \frac{\partial_{u} h_{0}(t-u)}{\gamma(u, t-u)} d u .
$$

The integral in this last expression converges as $\delta \rightarrow 0$, since $\gamma(u, t-u) \sim 2 \sqrt{u}$ and the $1 / \sqrt{u}$ singularity is integrable. Combining the contributions (5.20) and (5.21), then setting $\tau=t-u$ in the convolution integral, we find the result for the normal derivative of $C$ at the interface:

$$
\left.\frac{\partial C}{\partial N}\right|_{N=0}=-\frac{2 h_{0}(0)}{\sqrt{\pi} \gamma(t, 0)}-\frac{2}{\sqrt{\pi}} \int_{0}^{t} \frac{\partial_{\tau} h_{0}(\tau)}{\gamma(t-\tau, \tau)} d \tau .
$$

Copyright (c) by SIAM. Unauthorized reproduction of this article is prohibited. 
If the bulk and interface surfactant concentrations are in equilibrium initially, then $h_{0}(0)=0$, and the first term of (5.22) is zero.

The bulk-interface surfactant exchange term $\left.J_{0} \partial_{N} C\right|_{N=0}$ in (2.17) can now be evaluated using the convolution integral of (5.22) and, since no spatial mesh in the direction normal to the interface needs to be introduced to solve the transition layer equation, the method is referred to as mesh-free. In the implementation of the method described here, the convolution integral is evaluated directly at each time step by the trapezoidal rule. The time step for the interface update via the kinematic condition (2.3) and the evolution of surface surfactant concentration $\Gamma$ via (2.17) is by the forward Euler scheme. The spatial parameterization for the interface is by the Lagrangian fluid marker $\alpha=s_{0}$, so that the mesh-free method is spectrally accurate in space and first order in time.

A small time expansion has been developed to start the method, and details of this are given in [41]. Here we note that in the convolution integral, there is an integrable singularity as $\tau \rightarrow t^{-}$since from (5.19), $\gamma(t-\tau, \tau) \sim 2(t-\tau)^{1 / 2}$, and if the initial bulk and surface surfactant concentrations are not in equilibrium, $\partial_{\tau} h_{0}(\tau)$ has a $\tau^{-1 / 2}$ singularity as $\tau \rightarrow 0^{+}$.

6. Numerical results and discussion. In each of the three examples described here, the interface is initially circular, and the surface and bulk surfactant concentrations are in equilibrium. The first two examples are for an inviscid drop, or bubble, so that the viscosity ratio $\lambda=0$.

In the first example, the imposed flow is a pure strain or extensional flow $\boldsymbol{u}^{\infty}=$ $Q\left(x_{1},-x_{2}\right)$ with capillary number $Q=0.25$. Data are shown in Figure 6.1. In the

(a)

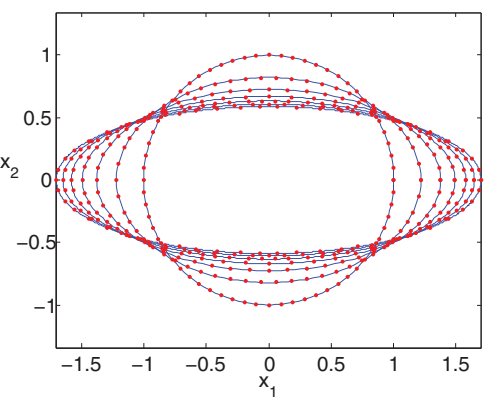

(c)

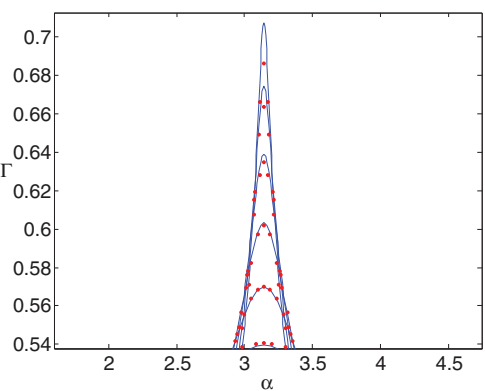

(b)

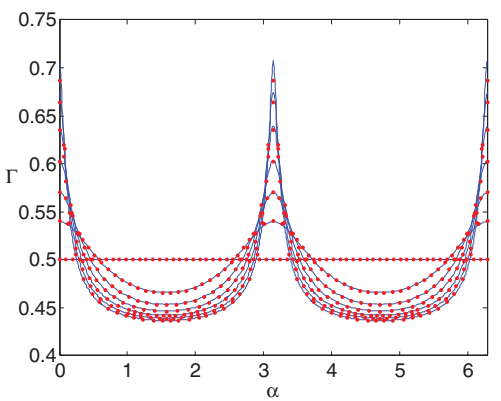

(d)

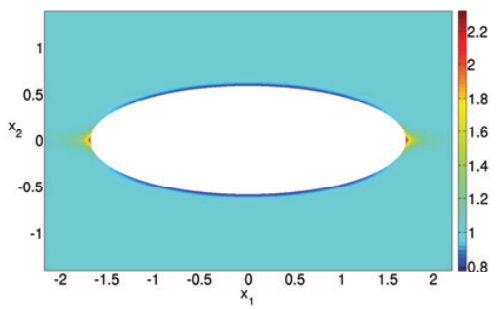

FIG. 6.1. An inviscid bubble is stretched in a pure strain with capillary number $Q=0.25$. Data found by the mesh-based method are indicated by lines, and data found by the mesh-free method are indicated by markers. (a) Interface profile at times $t=0,(0.5), 3.0$. (b) Surface surfactant concentration $\Gamma$ versus scaled arc length along the interface $\alpha$ at the same times. (c) Close-up of data of (b) near a bubble tip. (d) Bulk surfactant concentration $C$ at time $t=3.0$ with $P e=1,600$. 
three panels of Figure 6.1(a)-(c), the results of the mesh-based method of section 4 for solving the transition layer equation (2.14) are shown by lines, while the results of the mesh-free method of section 5 , with the bulk-interface surfactant exchange term in (2.17) given by the convolution integral of (5.22), are shown by markers.

In Figure 6.1(a) the interface profile is shown at times from $t=0.0$ to $t=3.0$ in increments of $\Delta t=0.5$. We noted in the introduction that in the limit of an inviscid drop, conformal mapping techniques imply that the profile is an ellipse for all time, but this result is not built into the solution method used here. A least squares fit of the data shows that the difference between the computed shapes and an ellipse is close to roundoff error for all times for both the mesh-based and mesh-free methods. The profile appears to be approaching a steady state at the final time $t=3.0$.

Figure 6.1(b) shows the surface concentration of surfactant $\Gamma$ versus scaled distance along the interface $\alpha \in[0,2 \pi)$, where $\alpha=0$ or $\alpha=\pi$ at the bubble tips or poles. This is shown at the same sequence of times as in Figure 6.1(a), and the initial equilibrium value of $\Gamma(\alpha, 0)=0.5$. In an extensional flow, the tangential component of the fluid velocity on the interface $\boldsymbol{u}_{s}$ is directed toward the bubble poles. In the absence of surface diffusion of surfactant, which is set to zero here (i.e., $P e_{s}=\infty$ ), and without the inclusion of surfactant solubility effects, this tends to decrease the surface surfactant concentration $\Gamma$ near the equator $(\alpha=\pi / 2,3 \pi / 2)$ and increase it near the bubble poles. This trend is seen in Figure 6.1(b), which shows a relatively broad and shallow minimum of $\Gamma$ around the equator and a narrow maximum near the poles. Figure 6.1(c) shows a close-up of the mesh-based (lines) and mesh-free (markers) data near the pole $\alpha=\pi$. It is in a neighborhood of this point that the agreement between the data shown for the mesh-based and mesh-free methods is worst, but it is found to improve if the number of interface fluid markers of the mesh-free method is increased.

Figure 6.1(d) shows the bulk surfactant concentration at the final time $t=3.0$. The ambient far-field bulk concentration $C=1$ was in equilibrium with the initial surface concentration $\Gamma(\alpha, 0)=0.5$ at $t=0$, since $K=1.0$. At later times, from the surface surfactant concentration data of Figure 6.1(b), the effect of surfactant solubility is such that along the broad and relatively flat equatorial section of the bubble the surface concentration is below equilibrium, and surfactant is drawn across the transition layer and enters the interface from the bulk. Conversely, at the highly curved bubble poles there is an excess of surface surfactant concentration, so that surfactant tends to leave the interface and enter the bulk flow. Other parameter values are $E=0.1$ and $J_{0}=1.0$.

The model for surfactant solubility effects that we use is based on an asymptotic reduction in the limit $P e \rightarrow \infty$ that keeps leading order terms only. As a consequence, as noted after (2.17), the bulk Péclet number is scaled out of the calculation, but a finite value of $P e$ must be introduced to display the data for $C$ in terms of the original unscaled spatial coordinates of the $x_{1}, x_{2}$-plane, and the transition layer width is scaled by $P e^{-1 / 2}$. In Figure $6.1(\mathrm{~d})$ the value $P e=1,600$ is chosen.

For all computations with the mesh-based method the number of ChebyshevLobatto points in the direction normal to the interface is fixed with $m=64$. The computations begin with a chosen number of $M$ points on the interface in the tangential or $\alpha$-direction. FFTs are used to track the interface profile and surface surfactant concentration $\Gamma$, and if the amplitude of Fourier modes near the Nyquist frequency increases above a threshold value, then $M$ is doubled. For the computations of Figure 6.1 by the mesh-based method, $M=128$ at the initial instant and $M=1024$ at the final time $t=3.0$. The time step is fixed at 0.001 , and the CPU time is 144 minutes. For computations with the mesh-free method, $\alpha=s_{0}$ is a Lagrangian fluid marker, 
(a)

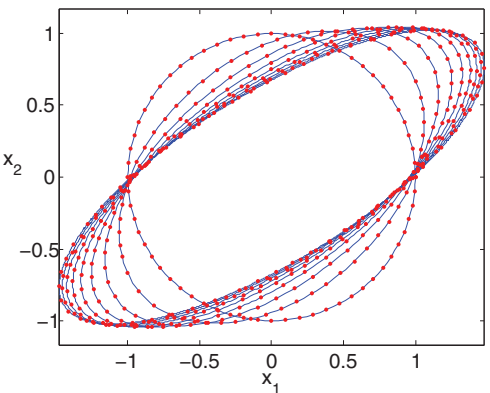

(c)

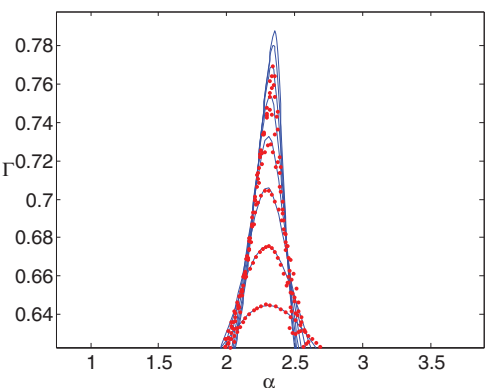

(b)

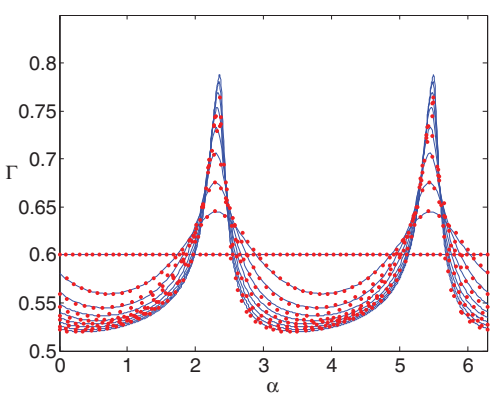

(d)

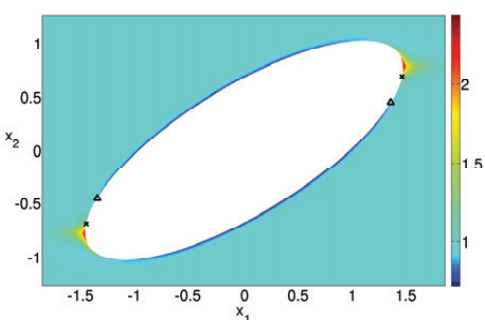

FIG. 6.2. An inviscid bubble is deformed by a simple shear with capillary number $G=0.5$. The results of the mesh-based method are indicated by lines, and results of the mesh-free method are indicated by markers. (a) Interface profile at times $t=0,(0.5), 4.0$. (b) Surface surfactant concentration $\Gamma$ versus scaled interface arc length $\alpha$ at the same times. (c) Close-up of data of (b) near a local maximum. (d) Bulk surfactant concentration $C$ at time $t=4.0$ with $P e=3,000$. Stagnation points on the interface are indicated by $\times$ (incoming interfacial flow) or $\triangle$ (outgoing interfacial flow).

and as time increases these tend to cluster near the high curvature bubble tips. In this case $M=128$ throughout the computation, the time step is 0.01 , and the CPU time is 31 minutes. For both methods, the bubble area is conserved with a relative error less than $10^{-8}$. All computations were performed with a $2.4 \mathrm{GHz}$, AMD Opteron model 180 processor.

The second example is of an inviscid bubble in a simple shear flow $\boldsymbol{u}^{\infty}=G\left(x_{2}, 0\right)$ with capillary number $G=0.5$. Data are shown in Figure 6.2. It is well known that the dynamics of a drop in simple shear and pure strain flows are quite different, and much of this is due to the nonzero vorticity of the simple shear. Figure 6.2(a) shows the interface profile from time $t=0.0$ to $t=4.0$ in increments of $\Delta t=0.5$. A least squares fit of the data shows that, as for the pure strain of the first example, the exact elliptical shape of the interface is maintained by both the mesh-based and the mesh-free methods with an error that is close to roundoff. The profile appears to be approaching a steady state at the final time $t=4.0$.

The surface concentration of surfactant $\Gamma$ is shown versus scaled arc length along the interface $\alpha \in[0,2 \pi)$ in Figure 6.2(b). The profile has less symmetry than is seen for a pure strain, it is now twofold as opposed to fourfold, and the maxima of $\Gamma$ that develop for $t>0$ are not symmetrical; there is a larger gradient of $\Gamma$ to the right of the peak than to the left in Figure 6.2(b). Figure 6.2(d) shows the bulk surfactant concentration $C$ at the final time $t=4.0$, plotted with $P e=3,000$. The initial surface surfactant concentration $\Gamma(\alpha, 0)=0.6$ was in equilibrium with the ambient bulk value $C(\boldsymbol{x}, 0)=1$, since $K=1.5$. Other parameter values are $E=0.1$ and $J_{0}=1.0$. 
Figure $6.2(\mathrm{~d})$ shows the location of four stagnation points on the interface at $t=4.0$. At the initial instant $t=0^{+}$, these are located symmetrically on the initial circular, equilibrium bubble shape. Then, as the bubble is deformed, each of the leftand right-hand pairs of stagnation points move toward each other simultaneously as the right-hand pair move upward and the left-hand pair move downward, moving toward unperturbed streamlines of the imposed shear flow. Throughout this part of the evolution $(t \in[0,4.0])$, the tangential fluid velocity on most of the interface is in the clockwise direction implied by the vorticity of the imposed flow.

Since the initial surface surfactant concentration is uniform and surface diffusion is set to zero $\left(P e_{s}=\infty\right)$, in the absence of solubility effects the surface surfactant concentration $\Gamma$ would be greatest where Lagrangian fluid markers congregate most closely during their motion along the interface. We find from the data that, with solubility effects included as described, these accumulation points of fluid markers still closely coincide with both the maxima of $\Gamma$ in Figure 6.2(b) and the maxima of $\left.C\right|_{N=0}$ at the interface in the transition layer, which are indicated by red (in color) in Figure 6.2(d). The accumulation points are close to but slightly displaced from the two stagnation points marked by a cross $(\times)$ in Figure $6.2(\mathrm{~d})$, at which the flow separates from the interface.

A separate computation carried out to $t=6.0$ with some extrapolation of the data suggests that the stagnation points on the interface coalesce at a later time $t \simeq 7.0$, to form a single stagnation point that moves some distance away from the interface. The tangential fluid velocity on the interface is then clockwise at all points, and the resulting interfacial motion is referred to as "tank-treading."

Agreement between the results of the mesh-based method (shown by lines) and the mesh-free method (shown by markers) is close, but from Figures 6.2(b) and (c) is at its worst near maxima of $\Gamma$ at later times. However, the results of the meshfree method approach those of the mesh-based method if the number of mesh-free Lagrangian markers is increased, as was also found in the example above for a pure strain.

In this example, the mesh-based computations begin with $M=128$ equally spaced points in the $\alpha$-direction, which increases to $M=512$ by the final time $t=4.0$. The time step is fixed at 0.001 , and the CPU time is 276 minutes. The computations with the mesh-free method have a constant number of $M=256$ Lagrangian fluid markers on the interface throughout, the time step is 0.0025 , and the CPU time is 332 minutes. In both methods, the bubble area is conserved with a relative error of less than $10^{-8}$.

The behavior seen in this and the next example is in broad agreement with the results and conclusions of Lee and Pozrikidis in [42]. They considered the influence of insoluble surfactant on 2D drops and bubbles in a 2D simple shear flow in the absence of solubility effects. Other differences are that in [42] the flow is bounded by moving, rigid, plane top and bottom boundaries in the $x_{2}$-direction, with periodic boundary conditions in the $x_{1}$-direction, and a different numerical approach is used that can incorporate nonzero Reynolds number effects. They note that, independently, both nonzero drop viscosity and insoluble surfactant tend to reduce the variation in tangential velocity around the interface and promote a tank-treading motion.

A nonzero interior or drop viscosity does this simply by opposing local shear. The mechanism for insoluble surfactant is based on the action of the Marangoni stress term $\nabla_{s} \sigma$ of (2.4) and the tendency of surfactant to immobilize the interface; see, for example, [11] for discussion of the influence of insoluble surfactant on a 3D drop in a simple shear flow. In an initial stage of deformation there is a relatively large variation in the tangential fluid speed $u_{s}=\boldsymbol{u}_{s} \cdot \boldsymbol{s}$ around the interface, which sets 
up a spatially nonuniform surface surfactant concentration. As seen in Figures 6.2(b) and (d) the concentration is low over much of the interface but is high near the highcurvature bubble ends. This sets up a surface tension gradient, or Marangoni stress, with high surface tension over much of the flat equatorial sections of the interface and low surface tension near the bubble ends. The direction of the Marangoni stress is such as to reduce the variation in tangential fluid velocity around the interface, which then tends to assume the direction implied by the vorticity of the imposed shear and promotes tank-treading.

The net influence of surfactant on drop deformation is the result of a combination of effects. By reducing surface tension, the presence of surfactant tends to reduce opposition to stretching of an interface and permits deformation that is induced by an imposed flow. However, in two dimensions the influence of surface gradients of surfactant and the direction of the resulting Marangoni stress just described act in a direction that tends to decrease drop length and reduce deformation; this holds for both a pure strain and a simple shear flow, while in three dimensions out-of-plain stress can lead to different dynamics. Moreover, the influence of surfactant solubility in the above examples tends to reduce that of Marangoni stress, as has been noted elsewhere [43]. Along the relatively large, flat, high-surface-tension regions of the drop midsections, the low surface surfactant concentration is below equilibrium with the far-field bulk concentration $C=1$, so that surfactant is drawn across the transition layer, from the bulk to the interface, thereby increasing the surface concentration and reducing surface tension. In a neighborhood of the surfactant accumulation points, at or near the high-curvature ends, the mechanism is reversed. The high surface concentration is above equilibrium with the far-field bulk concentration, and surfactant leaves the interface across the transition layer, tending to increase surface tension.

In both of the above examples, up to the time at the end of the computations there is a stagnation point on the interface that is either at (in Figure 6.1) or near (in Figure 6.2) a surfactant accumulation point, and the direction of the exterior flow tends to separate or is directed away from the interface. At later times, flow separation will continue to advect the relatively high local bulk surfactant concentration away from the interface in a plume. Our computations do not track this development here. In a simple shear flow, once the interface begins to tank-tread and the stagnation points move off the interface, the flow around the drop changes and is accompanied by a region of more or less closed fluid particle paths encircling the interface. This is discussed for insoluble surfactant in [42] and is seen in our next example with soluble surfactant.

Compared to the scenario of Figure 6.2, in this example we take a viscous drop with viscosity ratio $\lambda=1.2$ and an increased shear rate $G=1.0$. All other parameters remain unchanged (i.e., the initially circular drop is in equilibrium with $\Gamma(\alpha, 0)=0.6$, $K=1.5, E=0.1$, and $J_{0}=1.0$ ). The computations are taken up to $t=20.0$ and are shown for the mesh-based method. The number of points in the $\alpha$-direction is $M=128$ at $t=0$ and increases to $M=1024$ at $t=20.0$. The time step is fixed at 0.001 , and the CPU time is 47.6 hours. Data are shown in Figures 6.3 and 6.4.

Figure 6.3(a) shows the interface profile from time $t=0.0$ to time $t=20.0$ in increments of $\Delta t=1.0$. The profile elongates and reaches a maximum length at $t \simeq 9.0$ and then proceeds to retract. Figure 6.3(b) shows the surface surfactant concentration $\Gamma$ at a subset of the same times. Data for the tangential interface velocity (not shown) shows that this more viscous drop begins to tank-tread immediately at $t=0^{+}$. There is a brief interval around $t \in(1.5,3.5)$ when two pairs of stagnation points briefly appear on the interface, but the members of each pair remain close and 


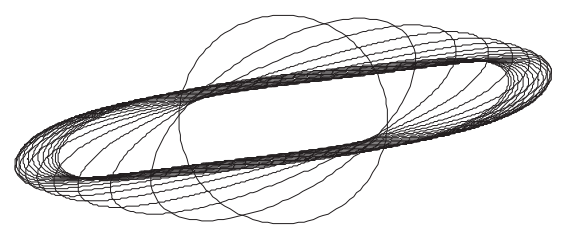

(a)

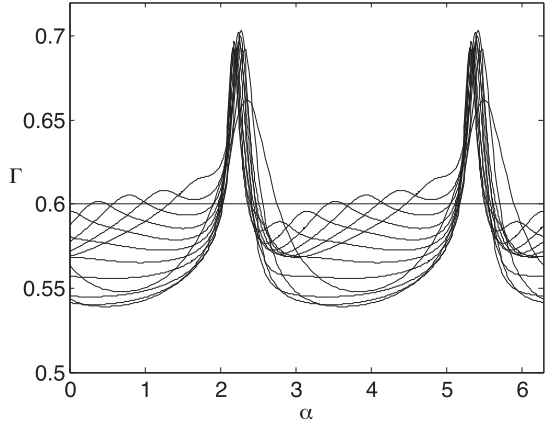

(b)

FIG. 6.3. A viscous drop with $\lambda=1.2$ is deformed by a simple shear flow with capillary number $G=1.0$. (a) Drop profile at times $t=0,(1.0), 20.0$. (b) Surface surfactant concentration $\Gamma$ versus scaled interface arc length $\alpha$ at times $t=0,(1.0), 4.0$ and $t=4.0,(2.0), 20.0$.
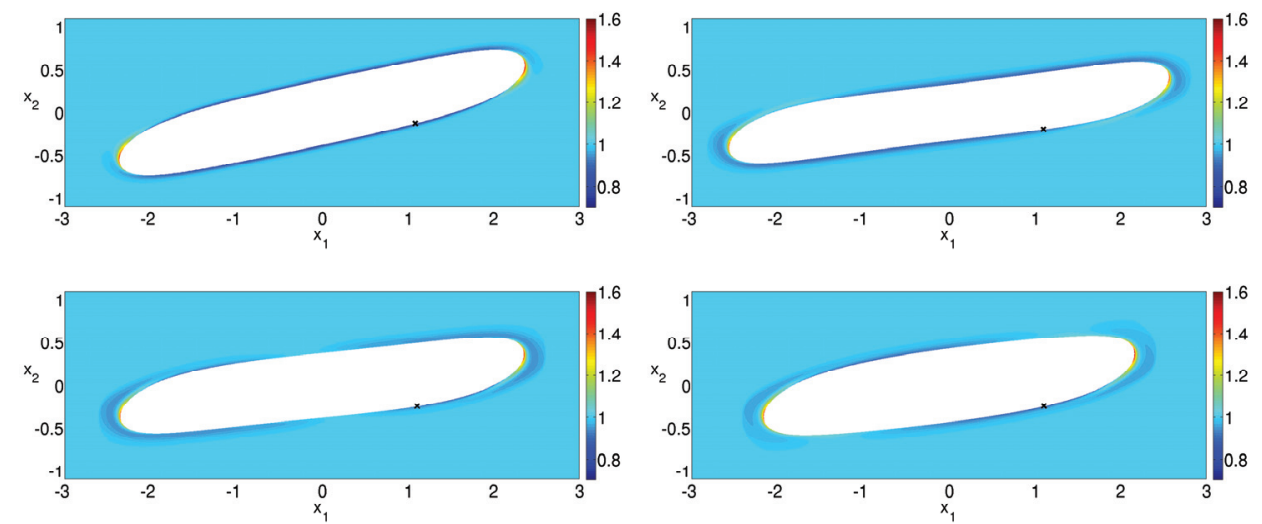

FIG. 6.4. Bulk surfactant concentration $C$ at times $t=5.0,(5.0), 20.0$ (left to right, top to bottom) for the same viscous drop of Figure 6.3 , with $\lambda=1.2$ and $G=1.0$. A cross $(\times)$ denotes the point $\alpha=0$ on the interface.

coalesce to leave the interface for all later times. Apart from this short interlude, the interface tank-treads.

In an initial stage of the evolution, for $t \in[0,4)$, the dynamics is broadly similar to that of the previous example (see Figure 6.2). A similar redistribution of surface surfactant concentration is set up, although the magnitude of its variation around the interface is slightly less, as is seen by comparison of the vertical scales of Figures $6.2(\mathrm{~b})$ and $6.3(\mathrm{~b})$. At later times the dynamics are markedly different, and some of this is attributable to the tank-treading motion of the interface and the nonseparated recirculation of the flow near the drop interface that accompanies it.

Figure 6.4 shows the bulk surfactant concentration $C$ at times $t=5.0,10.0,15.0$, and 20.0. It is useful to compare this with the surface surfactant concentration data for $\Gamma$ of Figure 6.3(b). At the time $t=5.0$ of the top-left panel of Figure 6.4, just after the initial stage of redistribution of $\Gamma$ is complete, a region of relatively low bulk concentration in the transition layer begins to be swept around the outside of the surfactant accumulation points near the drop ends. The bulk concentration in the transition layer becomes nonmonotone in the direction normal to the interface, which tends to enhance exchange of surfactant away from the interface to the bulk near 
the accumulation points, and this process continues for all later times. The result is a smearing out or reduction in spatial gradients of the surface and bulk surfactant concentrations around the interface.

The data for $\Gamma$ of Figure 6.3(b) show that at times $t>4.0$ the broad minima in $\Gamma$ over much of the long, flat midsections of the interface steadily increase, although there is still an appreciable spike in $\Gamma$ at the surface surfactant accumulation points. At the time $t=10.0$ of the top-right panel of Figure 6.4, the region of low bulk concentration in the transition layer has swept around just beyond the accumulation points at the drop ends. There is a small region beyond this where $C \simeq 1$ almost completely across the transition layer width, indicating that the surface and bulk concentrations are close to equilibrium. This is seen in Figure 6.3(b) as a relatively small-amplitude local maximum that breaks away from the neighboring spike in $\Gamma$ and then proceeds as a wave in the direction of increasing $\alpha$ until, at $t=20.0$, it is about to merge with the spike in $\Gamma$ at the far end of the drop. The same wave is also seen progressing along the flat midsections of the drop in the two lower panels of Figure 6.4 , where $t=15.0$ in the bottom-left panel and $t=20.0$ in the bottom-right panel. This is a result of the combined effects of tank-treading and surfactant solubility. The wave speed agrees with the data for the interface tangential fluid velocity, which also, for this slender highly deformed drop, is moderately close to that of the imposed shear.

We noted earlier that the drop profile in Figure 6.3(a) overshoots, or elongates, to a maximum length at $t \simeq 9.0$ and later retracts. The drop profile of Figure 6.4 for times $t>9.0$ suggests that the angle $\alpha^{\prime}$ of inclination of the drop between its major axis and the direction of the imposed flow may also be undergoing small-amplitude oscillations. However, the final time of our computations is not sufficient to determine details such as whether the oscillations are damped or sustained. The results of separate computations with constant surface tension confirm that, although features of the observed dynamics may be modified by surfactant effects, a qualitatively similar change in the inclination angle and overshoot of the drop deformation number $D^{\prime}$ occur in the absence of surfactant. We follow the usual definition that the drop deformation number is $D^{\prime}=(l-b) /(l+b)$, where $l$ and $b$ are respectively the maximum and minimum distances of the drop interface from its centroid.

It is known that a 3D, initially spherical, surfactant-free drop placed in a simple shear flow can approach a steady state via a sequence of damped oscillations or "wobble" in both $D^{\prime}$ and $\alpha^{\prime}$. This has been shown theoretically in the limit of small deformation and zero Reynolds number for surfactant-free drops [44, 45] and has been verified experimentally [46]. The theory predicts a relaxation time for approach to the steady state given by $\tau=a_{0} \mu^{i} / \sigma_{0}$, which is $\tau_{0}=\lambda$ in our dimensionless time units. This agrees with the apparent absence of any overshoot in the deformation number seen in the 2D inviscid example of Figure 6.2 but predicts a relaxation time of $\tau_{0}=1.2$ for the highly deformed $2 \mathrm{D}$ viscous drop example of Figures 6.3 and 6.4, where we estimate $\tau_{0} \simeq 5$ to 10 . The difference is not explained by simple adjustment of the relaxation time for reduced surface tension due to interfacial surfactant, since the surfactant-free value $\sigma_{0}=1$ and the initial equilibrium value $\sigma_{e q}=0.94$ are too close. Damped oscillations of $D^{\prime}$ and $\alpha^{\prime}$ on a time scale similar to that found here have been observed in two dimensions in [42]. On the face of it, surfactant-mediated immobilization of the interface may cause the dynamics of a surfactant-laden drop to be more like the dynamics of a capsule. Sustained oscillations in $D^{\prime}$ and $\alpha^{\prime}$ or "trembling" (also referred to as swinging or vacillating-breathing) have been shown to occur for models of a vesicle in a simple shear flow; see, for example, [47]. 
7. Conclusion. The main theme of this study is a Green's function-based method for the solution of a local transition or boundary layer equation that occurs in a model for the influence of soluble surfactant on two-phase flow in the infinite bulk Péclet number limit. This gives the Dirichlet-to-Neumann map that contains the influence of surfactant solubility on the dynamics in the form of a convolution integral over time, in which the spatial coordinates on the fluid interface appear parametrically. We refer to the method as mesh-free, since it determines the evolution of quantities such as the interface shape and surface surfactant concentration but requires no computation of the transition layer in the direction normal to the interface. The mesh-free method implemented here is spectrally accurate in space and first order in time.

Sample computations are shown that compare results of the mesh-free method with those of a more traditional, mesh-based method. A mesh-based method solves the transition layer equation directly, and we have chosen a mesh-based method that gives spectral accuracy in space and is second order in time.

The discussion toward the end of section 6 suggests some of the dynamics that may occur when surfactant effects are included. We hope to explore this in a later study.

The infinite Péclet number model and both the mesh-based and mesh-free numerical methods can in principle be generalized to three dimensions. In this study we have restricted attention to two dimensions, since, when the interior fluid is inviscid, conformal mapping techniques can also be applied and are known to give semianalytical results, such as elliptical interface profiles for all times and for all other parameter values. This gives a partial validation of our results and of the results for the mesh-free method in particular.

The results discussed in section 6 show that the CPU times for the mesh-based and mesh-free methods as implemented here are comparable. However, a significant speed-up of the mesh-free method can be achieved if the convolution integral in (5.22) can be evaluated by accelerated methods, such as those found in [48, 49]. Although a mesh-based method gives the bulk surfactant concentration throughout the transition layer automatically, it can also be found from the mesh-free method by calculation of an additional convolution integral, per (5.16).

We have considered examples where the bulk surfactant concentration outside the transition layer is spatially uniform. The method we use can also be extended to examples where this is spatially nonuniform but smooth. In the large bulk Péclet number limit, per the discussion above (2.15), the bulk concentration there is conserved or constant on particle paths to within order $O(\epsilon)$. The concentration outside the transition layer can be tracked by, for example, a semi-Lagrangian method [50, 51] using a relatively coarse grid. The solution adjacent to the interface as found by this method is then used to modify the far-field matching condition of (2.15) which influences the solution within the transition layer. In this case there is an additional computational expense, which has been avoided here, of calculating the fluid velocity on the computational grid in the exterior domain.

\section{REFERENCES}

[1] D. A. Edwards, H. Brenner, and D. T. Wasan, Interfacial Transport Processes and Rheology, Butterworth-Heinemann, Boston, 1991.

[2] M. R. Booty And M. Siegel, A hybrid numerical method for interfacial fluid flow with soluble surfactant, J. Comput. Phys., 229 (2010), pp. 3864-3883. 
[3] G. I. TAYLOR, The formation of emulsions in definable fields of flow, Proc. R. Soc. Lond. A, 146 (1934), pp. 501-523.

[4] H. P. Grace, Dispersion phenomena in high viscosity immiscible fluid systems and application of static mixers as dispersion devices in such systems, Chem. Engrg. Commun., 14 (1982), pp. 225-277.

[5] B. J. Bentley And L. G. Leal, A computer-controlled four-roll mill for investigations of particle and drop dynamics in two-dimensional linear shear flows, J. Fluid Mech., 167 (1986), pp. 219-240.

[6] R. A. DE Bruijn, Tipstreaming of drops in simple shear flows, Chem. Engrg. Sci., 48 (1993), pp. $277-284$.

[7] J. J. M. Janssen, A. Boon, And W. G. M. Agterof, Influence of dynamic interfacial properties on droplet breakup in simple shear flow, AIChE J., 40 (1994), pp. 1929-1939.

[8] J. J. M. Janssen, A. Boon, And W. G. M. Agterof, Influence of dynamic interfacial properties on droplet breakup in plane hyperbolic flow, AIChE J., 43 (1997), pp. 1436-1447.

[9] H. A. Stone And L. G. Leal, The effects of surfactants on drop deformation and breakup, J. Fluid Mech., 220 (1990), pp. 161-186.

[10] W. J. Milliken, H. A. Stone, and L. G. Leal, The effect of surfactant on the transient motion of Newtonian drops, Phys. Fluids, 5 (1993), pp. 69-79.

[11] I. B. Bazhlekov, P. D. Anderson, And H. E. H. Meijer, Numerical investigation of the effect of insoluble surfactants on drop deformation and breakup in simple shear flow, J. Colloid Interface Sci., 298 (2006), pp. 369-394.

[12] H. A. Stone, Dynamics of drop deformation and breakup in viscous fluids, in Annu. Rev. Fluid Mech. 26, Annual Reviews, Palo Alto, CA, 1994, pp. 65-102.

[13] J. EgGers, Nonlinear dynamics and breakup of free-surface flows, Rev. Modern Phys., 69 (1997), pp. 865-929.

[14] D. QuÉRÉ, Fluid coating on a fiber, in Annu. Rev. Fluid Mech. 31, Annual Reviews, Palo Alto, CA, 1999, pp. 347-384.

[15] O. A. BASARAn, Small-scale free surface flows with breakup: Drop formation and emerging applications, AIChE J., 48 (2002), pp. 1842-1848.

[16] C. D. Eggleton, T.-M. Tsai, and K. J. Stebe, Tip streaming from a drop in the presence of surfactants, Phys. Rev. Lett., 87 (2001), 048302.

[17] S. L. Anna And H. C. Mayer, Microscale tipstreaming in a microfluidic flow focusing device, Phys. Fluids, 18 (2006), 121512.

[18] S. Tanveer and G. Vasconcelos, Time-evolving bubbles in two-dimensional Stokes flow, J. Fluid Mech., 301 (1995), pp. 325-344.

[19] M. SiEgeL, Influence of surfactant on rounded and pointed bubbles in two-dimensional Stokes flow, SIAM J. Appl. Math., 59 (1999), pp. 1998-2027.

[20] N. I. Muskhelishvili, Some Basic Problems of the Mathematical Theory of Elasticity, P. Noordhoff, Groningen, The Netherlands, 1963.

[21] S. G. Mikhlin, Integral Equations and Their Applications to Certain Problems in Mechanics, Mathematical Physics and Technology, MacMillan, New York, 1964.

[22] L. G. Leal, Laminar Flow and Convective Transport Processes, Butterworth-Heinemann, Newton, MA, 1992.

[23] C.-H. Chang and E. I. Franses, Adsorption dynamics of surfactants at the air/water interface: A critical review of mathematical models, data, and mechanisms, Colloids Surfaces A, 100 (1995), pp. 1-45.

[24] H. Wong, D. Rumschitzki, and C. Maldarelli, On the surfactant mass balance at a deforming fluid interface, Phys. Fluids, 8 (1996), pp. 3203-3204.

[25] B. Ambravaneswaran and O. A. Basaran, Effects of insoluble surfactants on the nonlinear deformation and breakup of stretching liquid bridges, Phys. Fluids, 11 (1999), pp. 997-1015.

[26] R. V. Craster, O. K. Matar, and D. T. Papageorgiou, Pinchoff and satellite formation in surfactant covered viscous threads, Phys. Fluids, 14 (2002), pp. 1364-1376.

[27] Y.-N. Young, M. R. Booty, M. Siegel, And J. Li, Influence of surfactant solubility on the deformation and breakup of a bubble or capillary jet in a viscous fluid, Phys. Fluids, 21 (2009), 072105.

[28] L. Greengard, M. C. Kropinski, and A. Mayo, Integral equation methods for Stokes flow and isotropic elasticity in the plane, J. Comput. Phys., 125 (1996), pp. 403-414.

[29] M. C. A. Kropinski, An efficient numerical method for studying interfacial motion in twodimensional creeping flows, J. Comput. Phys., 171 (2001), pp. 479-508.

[30] M. C. A. Kropinski, Numerical methods for multiple inviscid interfaces in creeping flows, J. Comput. Phys., 180 (2002), pp. 1-24.

Copyright (c) by SIAM. Unauthorized reproduction of this article is prohibited. 
[31] M. C. A. Kropinski And E. Lushi, Efficient numerical methods for multiple surfactant-coated bubbles in a two-dimensional Stokes flow, J. Comput. Phys., 230 (2011), pp. 4466-4487.

[32] G. F. Carrier, M. Krook, and C. E. Pearson, Functions of a Complex Variable: Theory and Technique, Classics in Appl. Math. 49, SIAM, Philadelphia, 2005.

[33] W. E. Langlois, Slow Viscous Flow, MacMillan, New York, 1964.

[34] T. Hou, J. Lowengrub, And M. Shelley, Removing the stiffness from interfacial flows with surface tension, J. Comput. Phys., 114 (1994), pp. 312-338.

[35] A. I. VAn DE Vooren, A numerical investigation of the rolling up of vortex sheets, Proc. Roy. Soc. London Ser. A, 373 (1980), pp. 67-91.

[36] G. BAKER And A. NachBin, Stable methods for vortex sheet motion in the presence of surface tension, SIAM J. Sci. Comput., 19 (1998), pp. 1737-1766.

[37] L. N. Trefethen, Spectral Methods in MATLAB, Software Environ. Tools 10, SIAM, Philadelphia, 2000.

[38] A. F. H. WARD And L. TordaI, Time-dependence of boundary tensions of solutions. I. The role of diffusion in time-effects, J. Chem. Phys., 14 (1946), pp. 453-461.

[39] Y.-C. Liao, E. I. Franses, and O. A. Basaran, Computation of dynamic adsorption with adaptive integral, finite difference, and finite element methods, J. Colloid Interface Sci., 258 (2003), pp. 310-321.

[40] I. Stakgold, Green's Functions and Boundary Value Problems, 2nd ed., Wiley, New York, 1998.

[41] K. Xu, Computational Methods for Two-Phase Flow with Soluble Surfactant, Ph.D. thesis, Mathematical Sciences, New Jersey Institute of Technology, Newark, NJ, 2010.

[42] J. LeE And C. PozRikidis, Effect of surfactants on the deformation of drops and bubbles in Navier-Stokes flow, Comput. Fluids, 35 (2006), pp. 43-60.

[43] W. J. Milliken And L. G. Leal, The influence of surfactant on the deformation and breakup of a viscous drop: The effect of surfactant solubility, J. Colloid Interface Sci., 166 (1994), pp. $275-285$.

[44] R. G. Cox, The deformation of a drop in a general time-dependent fluid flow, J. Fluid Mech., 37 (1969), pp. 601-623.

[45] J. M. RAllison, Note on the time-dependent deformation of a viscous drop which is almost spherical, J. Fluid Mech., 98 (1980), pp. 625-633.

[46] S. Torza, R. G. Cox, And S. G. Mason, Particle motions in sheared suspensions XXVII. Transient and steady deformation and burst of liquid drops, J. Colloid Interface Sci., 38 (1972), pp. 395-411.

[47] P. M. Vlahovska, Y.-N. Young, G. Danker, And C. Misbah, Dynamics of a non-spherical microcapsule with incompressible interface in shear flow, J. Fluid Mech., 678 (2011), pp. 221-247.

[48] L. Greengard and J. Strain, A fast algorithm for the evaluation of heat potentials, Comm. Pure Appl. Math., 43 (1990), pp. 949-963.

[49] S. K. Veerapaneni and G. Biros, A high-order solver for the heat equation in $1 d$ domains with moving boundaries, SIAM J. Sci. Comput., 29 (2007), pp. 2581-2606.

[50] R. E. EWIng AND H. WANG, A summary of numerical methods for time-dependent advectiondominated partial differential equations, J. Comput. Appl. Math., 128 (2001), pp. 423-445.

[51] T. Nakamura, R. TANaka, T. Yabe, AND K. TAKizaWa, Exactly conservative semi-Lagrangian scheme for multi-dimensional hyperbolic equations with directional splitting technique, J. Comput. Phys., 174 (2001), pp. 171-207.

Copyright (c) by SIAM. Unauthorized reproduction of this article is prohibited. 